\title{
Service-Outage Capacity Maximization in Cognitive Radio for Parallel Fading Channels
}

\author{
Athipat Limmanee, Student Member, IEEE, Subhrakanti Dey, Senior Member, IEEE, \\ and Jamie S. Evans, Member, IEEE
}

\begin{abstract}
This paper focuses on a cognitive radio network consisting of a secondary user (SU) equipped with orthogonal frequency-division multiplexing (OFDM) technology able to access $N$ randomly fading frequency bands for transmitting delayinsensitive (e.g. data) as well as delay-sensitive (e.g. voice or video) data. Each band is licensed to a distinct delay-sensitive primary user (PU) interested in meeting a minimum rate guarantee for delay-sensitive services with a maximum allowable primary outage probability or a primary outage constraint (POC) . Typically, a PU is oblivious to the SU's existence and has its own power policy based on the channel side information (CSI) of its direct gain between the PU transmitter and the PU receiver only. Under the assumption that the SU knows PUs' power policies and CSI of the entire network, we solve the SU's ergodic capacity maximization problem subject to SU's average transmit power and outage probability constraints (SOC) and all POCs or the so-called service-outage based capacity maximization for $\mathrm{SU}$ with POCs. We use a rigorous probabilistic power allocation technique that allows us to derive optimal power policies applicable to both continuous and discrete fading channels. Also, a suboptimal power control policy is proposed in order to avoid the high computational complexity of the optimal policy when $N$ is large. Numerical results are presented to illustrate the performance of the power allocation algorithms.
\end{abstract}

Index Terms-Cognitive radio, power control, ergodic capacity, outage probability, parallel fading channels.

\section{INTRODUCTION}

A $\mathrm{S}$ wireless communication technology becomes more and more mature and widely used, the deployed radio spectrum becomes more crowded. A more efficient way to support the increasing demand in wireless services within the limited radio spectrum must therefore be conceived. Indeed, Federal Communications Commission (FCC) has reported that the spectrum scarcity problem arises because of the inflexible spectrum regulation policy rather than an actual physical spectrum drought. Most of the allocated spectrum is largely underutilized by licensed/primary users (PUs) and the utilization varies in time and space [1]. This motivated the concept of cognitive radio, first proposed in [2]. Cognitive radio (CR) technology offers a way to improve spectral efficiency by allowing unlicensed/secondary users (SUs) to exploit unused spectrum in dynamically changing environments, providing that a resultant interference from SUs does not degrade the

Paper approved by S. Roy, the Editor for Cross-Layer Wireless/Cognitive Radio Networks of the IEEE Communications Society. Manuscript received November 8, 2011; revised April 2, 2012.

The authors are with the Department of Electrical and Electronic Engineering, The University of Melbourne, VIC 3010, Australia (e-mail: a.limmanee@pgrad.unimelb.edu.au, \{sdey, jse\}@unimelb.edu.au).

Digital Object Identifier 10.1109/TCOMM.2012.101812.110747
Quality-of-Service (QoS) of the PU network to an unacceptable level. At present, there are three main approaches for CR to manage its resultant interference: interweave, overlay, and underlay [3]. In the underlay paradigm, which is the focus of this paper, CR users can share the spectrum or subcarrier as long as the QoS in primary system is guaranteed, regardless of the activity of the primary users.

It is well known that orthogonal frequency division multiplexing (OFDM) is regarded as a potential transmission technique for broadband wireless systems due to its high transmission efficiency, its robustness against inter-symbol interference in frequency selective channels, and especially due to its great flexibility in dynamically allocating transmission resources, making OFDM widely accepted as a promising candidate for future CR systems [4]. In OFDM-based CR system, SUs will have more alternatives to enhance its transmission efficiency, for instance, by using the spectrum gaps left by PUs [4], transmitting opportunistically through vacant subcarriers in the primary systems [5], or even sharing the subcarriers with PUs on the condition that the QoS of the primary system is guaranteed [6][7].

In this paper, we will focus on a transmit power allocation problem in an OFDM-based CR system within the underlay paradigm where an OFDM-based SU seeks a fundamental tradeoff between maximizing its own throughput with limited resources and minimizing the performance loss in the primary system. Resource allocation problems in OFDM-based CR systems have already attracted wide attention. In [8] and [9], it is shown that the water-filling power policy is the optimal transmission strategy to maximize ergodic channel capacity in a conventional OFDM system with a total average transmit power constraint across the sub-bands (TPC). This water-filling policy cannot be used when PUs' service quality constraints are taken into an account. In [6], the authors derived the solution of SU's instantaneous rate maximization problem with TPC and an individual interference power constraint on each subcarrier to protect the corresponding primary transmission. Then in [7], the authors proposed a new type of constraint to protect the PU QoS, called the 'rate loss constraint' (RLC), defined as the upper bound of the PU rate loss due to SU transmission. In [5], the authors derived the optimal power control policy for the SUs' sum ratemaximizing power allocation problem under the constraint that SU cross band interference incurred by the side-by-side PU frequency bands is limited. However, if the PUs are engaged in transmission of delay-sensitive information, the PU outage 
probability constraint becomes a more suitable measure for protecting a delay-sensitive primary user in [10], [11] where the optimal power allocation problems for the secondary ergodic and outage capacity maximization problem with a primary outage constraint were addressed and later further extended in [12]. Finally, an effective capacity based delay QoS constraint on the SU and a PU outage constraint was considered in [13], where the authors propose a variable-rate variable-power based MQAM scheme to solved the associated optimization problems with full CSI.

In this paper, we consider a CR network where an OFDMbased SU operates in an orthogonal frequency division multiple access (OFDMA) based primary system. The SU aims to transmit both delay-sensitive and delay-insensitive information (such as integrated voice/video and packet data) over $N$ subcarriers of the OFDMA-based primary system. Each subcarrier is licensed to an individual PU that wishes to maintain a basic rate with a certain outage probability. In other words, this paper solves the SU's ergodic capacity (SEC) maximization problem under an SU's outage probability constraint (SOC), $N$ PUs' outage probability constraints (POCs), and an SU's average transmit power constraint (ATPC). This problem is closely related to the 'service-outage capacity problem in parallel fading channel' [14] in the sense that if all $N$ POCs are discarded from our problem, the two problem become exactly the same. The idea is that as soon as the service quality of the delay-sensitive information (voice) is ensured by a guaranteed outage probability, any excess rate can be used to delay-insensitive information (data) in a best-effort fashion. The problem also extends the result in [15], where only one single frequency band is considered. Furthermore, this paper addresses the relationship between the feasibility of the SEC maximization problem and the problem of SU's outage probability (SO) minimization subject to all $N$ POCs and an ATPC. This SO problem was initially addressed in [11] for $N=1$. In our paper, the result of [11] is generalized to the case when $N>1$. Under the assumption that the PU in each subcarrier has a transmission policy that is based on its own channel between its corresponding primary transmitter and receiver only and that the SU has knowledge of all PUs' transmission policies as well as the full channel state information (CSI) of the entire network, both SEC and SO problems are solved by using a rigorous 'probabilistic power allocation' technique, originally proposed in [16]. This method allows us to treat the optimal power allocation problem as a convex optimization problem and renders our power allocation results applicable to both continuous as well as discrete fading channels. However, the optimal power control for the SEC problem results in a high computational complexity (exponential in $N$ ) when the number of sub-carriers, $N$ is large. Thus motivated, we also propose a suboptimal power control policy with a reduced real-time computational complexity to alleviate this problem. Numerical studies illustrate the performance of the optimal power policies and demonstrate that our proposed suboptimal policy is not only computationally efficient, but also satisfies the SOC and all POCs incurring a small SU ergodic capacity loss.

The rest of paper is organized as follows. Section II presents the system model. We formulate SEC maximization problems in Section III. Section IV demonstrates the derivation on the optimal solution for the SEC problem, discusses the feasibility of SEC problem and its relationship with the extended SO minimization problem, and proposes a low-complexity suboptimal power scheme for the SEC maximization problem. Illustrative numerical results are provided in Section $\mathrm{V}$ followed by some concluding remarks in Section VI.

Notation: $\frac{\partial y}{\partial x^{*}}$ denotes the derivative of $y$ over $x$ evaluated at $x=x^{*}$. $\prec, \preceq$ denote componentwise strict inequality and componentwise inequality in $\mathbf{R}^{N}$, respectively. $\langle\mathbf{x}\rangle=\sum_{i=1}^{N} x_{i}$.

\section{System Model}

We consider a cognitive radio environment with $N$ primary transmitter-receiver pairs (PT-PR) and a single OFDMbased secondary transmitter-receiver pair (ST-SR). The SU can access all $N$ frequency bands of which the $i$-th band is licensed to the $i$-th $\mathrm{PU}\left(\mathrm{PU}_{i}\right)$ for $i \in\{1,2, \ldots, N\}$ in an OFDMA-based primary system. All channels involved in this cognitive radio network are assumed to be block fading additive white Gaussian noise (BF-AWGN) channels [16]. The instantaneous channel power gains in the $i$-th subchannel for the link $\mathrm{PT}_{i}-\mathrm{PR}_{i}, \mathrm{ST}-\mathrm{SR}, \mathrm{PT}_{i}-\mathrm{SR}$, and $\mathrm{ST}_{-} \mathrm{PR}_{i}$ are denoted by $g_{i}, h_{i}, \alpha_{i}$, and $\beta_{i}$, respectively. Let $\nu_{i} \triangleq\left[g_{i}, h_{i}, \alpha_{i}, \beta_{i}\right]$ and $\nu \triangleq\left[\nu_{1}, \nu_{2}, \ldots, \nu_{N}\right]$ represent the combined channel state vector. The vector fading process $\nu$ is presumed to be stationary and ergodic with a cumulative density function $F(\boldsymbol{\nu})$. The additive noises at PR and SR in $i$-th subchannel are assumed to be independent Gaussian random variables with zero mean and variance $N_{0}$. We assume that SU transmitter has full CSI of $\boldsymbol{\nu}$, i.e. all channel gains in the network, while the $i$-th PU has full CSI for the direct channel power gain $g_{i}$ between $\mathrm{PT}_{i}$ and $\mathrm{PR}_{i}$ only.

Remark 1: In our problem formulation, we do not allow the primary users to share the $N$ channel bands to avoid PUs causing interference to each other. Consideration of PU generated mutual interference or an appropriate scheduling policy that allocates each band to a distinct PU in every fading block will render our problem formulation rather complex and is beyond the scope of the current paper. Note however that it is easy to extend the results of the current paper to the case where each primary user has a distinct set of subcarriers (so that the primary users do not cause interference to each other) that it can use, although this will result in an increased complexity for the power allocation problem.

Remark 2: Note that the assumption of full CSI at the ST of all channels is not realistic for a practical cognitive radio system, as much as in any existing wireless communication systems. In particular, obtaining full channel information of the SU-PR channels and PT-PR channels may be difficult. In recent literature however, some practical schemes have been suggested for obtaining such information at the ST in [17]. For ST-PR channels, it is suggested that the ST can estimate this gain by measuring received power of signals transmitted by the PR and under the assumption of channel reciprocity and that $\mathrm{ST}$ knows the PR transmission power. For PT-PR channels, various suggestions have been made including that of eavesdropping on PR feedback to PT [18], 
and receiving feedback from a cooperative SU node employed near the PR [19], while information about ST-SR channels can be obtained via classical channel feedback and training schemes. Furthermore, power allocation for the secondary user's ergodic capacity maximization under average transmit power and average interference (peak interference) (at the primary receiver) constraints in a spectrum sharing scenario with quantized CSI (or limited feedback) has been investigated in [20] ([21]). Design and analysis of such limited feedback based design for the service-outage considered in this current submission is a considerably much harder problem and will be investigated in future work. The results obtained in the current paper based on full CSI will serve as a benchmark for any such future results based on partial or imperfect CSI.

Remark 3: We have addressed the above issue of possible unavailability of full CSI at the ST further by illustrating the effect of imperfect or partial CSI at the ST in terms of SU ergodic capacity loss. We have carried out a sensitivity analysis with respect to noisy estimated CSI of the PT-PR channels $g_{i}, i=1,2, \ldots, N$. For further details, see Figure 6 in the Numerical Results Section.

Our main focus is on the service-outage based power allocation problem which combines the concepts of ergodic capacity and outage capacity. Let $P_{p i}\left(g_{i}\right)$ represent $\mathrm{PU}_{i}$ 's power strategy as $\mathrm{PU}_{i}$ 's power policy is assumed to be determined by the direct channel power gain $g_{i}$ between $\mathrm{PT}_{i}$ and $\mathrm{PR}_{i}$ only, due to a common assumption that the PUs are generally oblivious to the presence of the SU. $\mathbf{P}_{s}(\boldsymbol{\nu})=\left[P_{s 1}(\boldsymbol{\nu}), \ldots, P_{s N}(\boldsymbol{\nu})\right]$ denotes SU's power allocation strategy as a function of the channel state $\boldsymbol{\nu}$, where $P_{s i}(\boldsymbol{\nu})$ is the transmission power for the $i$-th band. The instantaneous transmission rates $r_{p i}$ for the $i$-th $\mathrm{PU}$ and $r_{s}$ for the SU can be defined as $r_{p i}\left(\boldsymbol{\nu}, P_{s i}(\boldsymbol{\nu})\right)=\log \left(1+\frac{g_{i} P_{p i}\left(g_{i}\right)}{\beta_{i} P_{s i}(\boldsymbol{\nu})+N_{0}}\right)$ and $r_{s}(\boldsymbol{\nu}, \mathbf{P}(\boldsymbol{\nu}))=\sum_{i=1}^{N} r_{s i}\left(\boldsymbol{\nu}, P_{s i}(\boldsymbol{\nu})\right)=\sum_{i=1}^{N} \log \left(1+\frac{h_{i} P_{s i}(\boldsymbol{\nu})}{\alpha_{i} P_{p i}\left(g_{i}\right)+N_{0}}\right)$. Note that we drop the constant $\frac{1}{2}$ and use natural logarithm for simplicity and $g_{i}$ is in fact an element of $\boldsymbol{\nu}$. Let $r_{p i}^{0}$ and $r_{s}^{0}$ denote the service rates of $\mathrm{PU}_{i}$ and the SU, respectively, and $\mathbf{r}_{p}^{0}=\left[r_{p 1}^{0}, r_{p 2}^{0}, \ldots, r_{p N}^{0}\right]$. In this work, we assume that each PU adopts an ON-OFF power control policy, i.e. $P_{p i}\left(g_{i}\right)=P_{c i}$ if $g_{i} \geq g_{T, i}=\frac{\left(e^{r_{p i}^{0}}-1\right) N_{0}}{P_{c i}}$, i.e. $g_{i}$ is $\operatorname{good}$ enough to support $r_{p i}^{0}$, and $P_{p i}\left(g_{i}\right) \stackrel{P_{c i}}{=} 0$ otherwise. In the absence of any $\mathrm{SU}$ transmission, $\mathrm{PU}_{i}$ 's outage probability becomes $\epsilon_{p i}^{c}=\operatorname{Pr}\left\{\log \left(1+\frac{g_{i} P_{c i}}{N_{0}}\right)<r_{p i}^{0}\right\}$. Therefore, when the $\mathrm{SU}$ is active, $\mathrm{PU}_{i}$ 's communication is protected as long as $\epsilon_{p i}^{c} \leq \operatorname{Pr}\left\{\log \left(1+\frac{g_{i} P_{c i}}{\beta_{i} P_{s i}(\boldsymbol{\nu})+N_{0}}\right)<r_{p i}^{0}\right\} \leq \epsilon_{p i}$, where $\epsilon_{p i}$ is the outage probability threshold of $\mathrm{PU}_{i}$. Define $\epsilon_{p}=$ $\left[\epsilon_{p 1}, \ldots, \epsilon_{p N}\right]$ and let $\epsilon_{s}$ represent the SU outage probability threshold. Note that the results in this paper can be extended to any other transmission power policy for the primary users as long as this policy is known to the SU.

\section{PROBlem Formulation}

The service-outage problem in this paper can be formulated as follows:

$$
\begin{array}{ll}
\max _{\mathbf{P}_{s}(\boldsymbol{\nu}) \succeq 0} & E\left[\sum_{i=1}^{N} r_{s i}\left(\boldsymbol{\nu}, P_{s i}(\boldsymbol{\nu})\right)\right] \\
\text { s.t. } & \text { (a) } E\left[\sum_{i=1}^{N} P_{s i}(\boldsymbol{\nu})\right] \leq P_{a v}, \\
& \text { (b) } \operatorname{Pr}\left\{\sum_{i=1}^{N} r_{s i}\left(\boldsymbol{\nu}, P_{s i}(\boldsymbol{\nu})\right)<r_{s}^{0}\right\} \leq \epsilon_{s}, \\
& \text { (c) } \operatorname{Pr}\left\{r_{p i}\left(\boldsymbol{\nu}, P_{s i}(\boldsymbol{\nu})\right)<r_{p i}^{0}\right\} \leq \epsilon_{p i}, \forall i
\end{array}
$$

It was shown in [14] and [16] that for such outage-based optimization problems, a deterministic power allocation policy is not optimal in general, especially for discrete fading channel distributions. Similar to [14] we show that (1) can be solved by using a probabilistic power allocation technique, i.e. by treating $\mathbf{P}_{s}(\boldsymbol{\nu})$ as a probabilistic power allocation scheme with a conditional probability density function (PDF) $f_{\mathbf{P}_{s} \mid \boldsymbol{\nu}}\left(\mathbf{p}_{s} \mid \boldsymbol{\nu}\right)$, having transmit power $P_{s i}(\boldsymbol{\nu})$ through $i$-th subchannel with conditional PDF $f_{P_{s i} \mid \boldsymbol{\nu}}\left(p_{s i} \mid \boldsymbol{\nu}\right)$. Note also that such a probabilistic power allocation policy helps transform the above optimization problem (1) into a standard convex optimization problem following similar lines as in [14]. $\mathbf{p}_{s}(\boldsymbol{\nu})=\left[p_{s 1}(\boldsymbol{\nu}), \ldots, p_{s N}(\boldsymbol{\nu})\right]$ where $p_{s i}(\boldsymbol{\nu})$ indicates a deterministic power allocation policy for the $i$-th subchannel. With the assumptions of ergodicity of the fading channels and perfect CSI at the SU transmitter, we have

$$
\begin{aligned}
& E\left[r_{s}\left(\boldsymbol{\nu}, \mathbf{P}_{s}(\boldsymbol{\nu})\right)\right]=\iint r_{s}\left(\boldsymbol{\nu}, \mathbf{p}_{s}(\boldsymbol{\nu})\right) f_{\mathbf{P}_{s} \mid \boldsymbol{\nu}}\left(\mathbf{p}_{s} \mid \boldsymbol{\nu}\right) d \mathbf{p}_{s}(\boldsymbol{\nu}) d F(\boldsymbol{\nu}) \\
& E\left[\left\langle\mathbf{P}_{s}(\boldsymbol{\nu})\right\rangle\right]=\iint\left\langle\mathbf{p}_{s}(\boldsymbol{\nu})\right\rangle f_{\mathbf{P}_{s} \mid \boldsymbol{\nu}}\left(\mathbf{p}_{s} \mid \boldsymbol{\nu}\right) d \mathbf{p}_{s}(\boldsymbol{\nu}) d F(\boldsymbol{\nu}) \\
& \operatorname{Pr}\left\{r_{s}\left(\boldsymbol{\nu}, \mathbf{P}_{s}(\boldsymbol{\nu})\right)<r_{s}^{0}\right\}= \\
& \iint 1\left(r_{s}\left(\boldsymbol{\nu}, \mathbf{p}_{s}(\boldsymbol{\nu})\right)<r_{s}^{0}\right) f_{\mathbf{P}_{s} \mid \boldsymbol{\nu}}\left(\mathbf{p}_{s} \mid \boldsymbol{\nu}\right) d \mathbf{p}_{s}(\boldsymbol{\nu}) d F(\boldsymbol{\nu}) \\
& \operatorname{Pr}\left\{r_{p i}\left(\boldsymbol{\nu}, P_{s i}(\boldsymbol{\nu})\right)<r_{p i}^{0}\right\}= \\
& \iint 1\left(r_{p i}\left(\boldsymbol{\nu}, p_{s i}(\boldsymbol{\nu})\right)<r_{p i}^{0}\right) f_{P_{s i} \mid \boldsymbol{\nu}}\left(p_{s i} \mid \boldsymbol{\nu}\right) d p_{s i}(\boldsymbol{\nu}) d F(\boldsymbol{\nu})
\end{aligned}
$$

where $f_{\mathbf{P}_{s} \mid \boldsymbol{\nu}}\left(\mathbf{p}_{s} \mid \boldsymbol{\nu}\right) \geq 0, f_{P_{s i} \mid \boldsymbol{\nu}}\left(p_{s i} \mid \boldsymbol{\nu}\right) \geq 0$, and $\int f_{\mathbf{P}_{s} \mid \boldsymbol{\nu}}\left(\mathbf{p}_{s} \mid \boldsymbol{\nu}\right) d \mathbf{p}_{s}(\boldsymbol{\nu})$ and $\int f_{P_{s i} \mid \boldsymbol{\nu}}\left(p_{s i} \mid \boldsymbol{\nu}\right) d p_{s i}(\boldsymbol{\nu})$ are 1 for all $i$, and $l(A)$ denote the indicator function taking value 1 when the event $A$ is true, and 0 otherwise.

Adapting a technique similar to the one used in [14], we define the 4 weighting functions and 4 corresponding deterministic power schemes for each of the subchannels as shown in (3) and (4).

$$
\begin{aligned}
& p_{1 i}(\boldsymbol{\nu})=E\left[P_{s i}(\boldsymbol{\nu}) \mid r_{p i}\left(\boldsymbol{\nu}, p_{s i}(\boldsymbol{\nu})\right) \geq r_{p i}^{0},\right. \\
& \left.\sum_{i=1}^{N} r_{s i}\left(\boldsymbol{\nu}, p_{s i}(\boldsymbol{\nu})\right) \geq r_{s}^{0}, \boldsymbol{\nu}\right] \\
& p_{2 i}(\boldsymbol{\nu})=E\left[P_{s i}(\boldsymbol{\nu}) \mid r_{p i}\left(\boldsymbol{\nu}, p_{s i}(\boldsymbol{\nu})\right)<r_{p i}^{0},\right. \\
& \left.\sum_{i=1}^{N} r_{s i}\left(\boldsymbol{\nu}, p_{s i}(\boldsymbol{\nu})\right) \geq r_{s}^{0}, \boldsymbol{\nu}\right] \\
& p_{3 i}(\boldsymbol{\nu})=E\left[P_{s i}(\boldsymbol{\nu}) \mid r_{p i}\left(\boldsymbol{\nu}, p_{s i}(\boldsymbol{\nu})\right) \geq r_{p i}^{0},\right. \\
& \left.\sum_{i=1}^{N} r_{s i}\left(\boldsymbol{\nu}, p_{s i}(\boldsymbol{\nu})\right)<r_{s}^{0}, \boldsymbol{\nu}\right] \\
& p_{4 i}(\boldsymbol{\nu})=E\left[P_{s i}(\boldsymbol{\nu}) \mid r_{p i}\left(\boldsymbol{\nu}, p_{s i}(\boldsymbol{\nu})\right)<r_{p i}^{0},\right. \\
& \left.\sum_{i=1}^{N} r_{s i}\left(\boldsymbol{\nu}, p_{s i}(\boldsymbol{\nu})\right)<r_{s}^{0}, \boldsymbol{\nu}\right]
\end{aligned}
$$

Note that the weighting function $w_{k i}(\boldsymbol{\nu})$ represents the probability of using the deterministic power strategy $p_{k i}(\boldsymbol{\nu})$, which is computed as the expectation of the probabilistic power control $P_{s i}(\boldsymbol{\nu})$ within the set that corresponds to $w_{k i}(\boldsymbol{\nu})$. 


$$
\begin{aligned}
& w_{1 i}(\boldsymbol{\nu})=\operatorname{Pr}\left\{r_{p i}\left(\boldsymbol{\nu}, P_{s i}(\boldsymbol{\nu})\right) \geq r_{p i}^{0}, \sum_{i=1}^{N} r_{s i}\left(\boldsymbol{\nu}, P_{s i}(\boldsymbol{\nu})\right) \geq r_{s}^{0} \mid \boldsymbol{\nu}\right\} \\
& w_{2 i}(\boldsymbol{\nu})=\operatorname{Pr}\left\{r_{p i}\left(\boldsymbol{\nu}, P_{s i}(\boldsymbol{\nu})\right)<r_{p i}^{0}, \sum_{i=1}^{N} r_{s i}\left(\boldsymbol{\nu}, P_{s i}(\boldsymbol{\nu})\right) \geq r_{s}^{0} \mid \boldsymbol{\nu}\right\} \\
& w_{3 i}(\boldsymbol{\nu})=\operatorname{Pr}\left\{r_{p i}\left(\boldsymbol{\nu}, P_{s i}(\boldsymbol{\nu})\right) \geq r_{p i}^{0}, \sum_{i=1}^{N} r_{s i}\left(\boldsymbol{\nu}, P_{s i}(\boldsymbol{\nu})\right)<r_{s}^{0} \mid \boldsymbol{\nu}\right\} \\
& w_{4 i}(\boldsymbol{\nu})=\operatorname{Pr}\left\{r_{p i}\left(\boldsymbol{\nu}, P_{s i}(\boldsymbol{\nu})\right)<r_{p i}^{0}, \sum_{i=1}^{N} r_{s i}\left(\boldsymbol{\nu}, P_{s i}(\boldsymbol{\nu})\right)<r_{s}^{0} \mid \boldsymbol{\nu}\right\}
\end{aligned}
$$

Let $w_{a}(\boldsymbol{\nu})=\operatorname{Pr}\left\{\sum_{i=1}^{N} r_{s i}\left(\boldsymbol{\nu}, P_{s i}(\boldsymbol{\nu})\right) \geq r_{s}^{0} \mid \boldsymbol{\nu}\right\}$. It follows that $w_{2 i}(\boldsymbol{\nu})=w_{a}(\boldsymbol{\nu})-w_{1 i}(\boldsymbol{\nu})$ and $w_{4 i}(\boldsymbol{\nu})=1-w_{a}(\boldsymbol{\nu})-$ $w_{3 i}(\boldsymbol{\nu})$ for all $i$. Also, let $X_{w_{k i}^{*}}$ be a Bernoulli random variable which is equal to 1 with probability $w_{k i}^{*}$ and 0 for otherwise. We now have the following Lemma the detailed proof of which can be found in Appendix A. Essentially, given an arbitrary feasibly policy, one can construct a randomized policy as stated in the Lemma below and show that this policy satisfies all the constraints and achieves an equal or better rate than the arbitrary feasible policy.

Lemma 1: There exists an optimum solution $\mathbf{P}_{s}^{*}(\boldsymbol{\nu})$ of problem (1) of the form $P_{s i}^{*}(\boldsymbol{\nu})=\sum_{k=1}^{4} X_{w_{k i}^{*}}(\boldsymbol{\nu}) p_{k i}^{*}(\boldsymbol{\nu})$ where

- (1) $\left[\sum_{i=1}^{N} r_{s i}\left(\boldsymbol{\nu}, \frac{w_{1 i}^{*}}{w_{1 i}^{*}+w_{2 i}^{*}} p_{1 i}^{*}+\frac{w_{2 i}^{*}}{w_{1 i}^{*}+w_{2 i}^{*}} p_{2 i}^{*}\right)-r_{s}^{0}\right] \geq 0$, $w_{1 i}^{*}(\boldsymbol{\nu})\left[\frac{1}{\beta_{i}}\left(\frac{g_{i} P_{p i}\left(g_{i}\right)}{e^{r_{p i}^{0}}-1}-N_{0}\right)^{+}-p_{1 i}^{*}(\boldsymbol{\nu})\right]+$ $w_{3 i}^{*}(\boldsymbol{\nu})\left[\frac{1}{\beta_{i}}\left(\frac{g_{i} P_{p i}\left(g_{i}\right)}{e^{r_{p i}^{0}}-1}-N_{0}\right)^{+}-p_{3 i}^{*}(\boldsymbol{\nu})\right] \geq 0$,

(3) $w_{1 i}^{*}(\boldsymbol{\nu})+w_{2 i}^{*}(\boldsymbol{\nu})=w_{1 j}^{*}(\boldsymbol{\nu})+w_{2 j}^{*}(\boldsymbol{\nu})=w_{a}^{*}(\boldsymbol{\nu})$,

(4) $\sum_{k=1}^{4} w_{k i}^{*}(\boldsymbol{\nu})=1$,

(5) $E\left[w_{1 i}^{*}(\boldsymbol{\nu})+w_{2 i}^{*}(\boldsymbol{\nu})\right]=E\left[w_{a}^{*}(\boldsymbol{\nu})\right] \geq 1-\epsilon_{s}$,

(6) $E\left[w_{1 i}^{*}(\boldsymbol{\nu})+w_{3 i}^{*}(\boldsymbol{\nu})\right] \geq 1-\epsilon_{p i}$,

(7) $E\left[\sum_{i=1}^{N} \sum_{k=1}^{4} w_{k i}^{*}(\boldsymbol{\nu}) p_{k i}^{*}(\boldsymbol{\nu})\right] \leq P_{a v}$.

for all $i, j \in\{1,2, \ldots N\}$ and $i \neq j$.

Using Lemma 1, (1) can be rewritten as shown in (5)

It can be shown that (5) is a convex optimization problem. The proof of convexity can be found in Appendix B. For convenience, we define $\mathbf{p}_{r s}(\boldsymbol{\nu})=\left[p_{r s, 1}(\boldsymbol{\nu}), \ldots, p_{r s, N}(\boldsymbol{\nu})\right]$ with $p_{r s, i}(\boldsymbol{\nu})=\left(\mu(\boldsymbol{\nu})-\frac{\alpha_{i} P_{p i}\left(g_{i}\right)+N_{0}}{h_{i}}\right)^{+}$where $\mu(\boldsymbol{\nu})$ satisfies $\sum_{i=1}^{N} r_{s i}\left(\boldsymbol{\nu}, p_{r s, i}(\boldsymbol{\nu})\right)=r_{s}^{0}$, and is derived by minimizing $\sum_{i=1}^{N} p_{r s, i}(\boldsymbol{\nu})$ subject to $\sum_{i=1}^{N} r_{s i}\left(\boldsymbol{\nu}, p_{r s, i}(\boldsymbol{\nu})\right) \geq r_{s}^{0}$. Note that $\mu(\boldsymbol{\nu})$ is obviously strictly positive. Also, define $p_{r p, i}(\boldsymbol{\nu})=$ $\frac{1}{\beta_{i}}\left(\frac{g_{i} P_{p i}\left(g_{i}\right)}{e^{r_{p i}^{0}}-1}-N_{0}\right)^{+}$so that $r_{p i}\left(\boldsymbol{\nu}, p_{r p, i}(\boldsymbol{\nu})\right)=r_{p i}^{0}$.

\section{Service-outage Capacity Maximization PROBLEM WITH $N$ POCS}

In this section, we will focus on solving the optimization Problem (1) by a functional optimization technique similar to [14] and conclude with results regarding the feasibility of this problem. First, note that the Lagrangian for (5) can be written as shown in (6) where $\lambda, s_{a}, s_{i}, q(\boldsymbol{\nu}), u_{i}(\boldsymbol{\nu}), \gamma_{i}(\boldsymbol{\nu})$, and $\eta_{i}(\boldsymbol{\nu})$ are the nonnegative Lagrange multipliers corresponding to constraints (5a) to $(5 \mathrm{~g})$. From the associated (necessary and sufficient) KKT conditions, we have for $i=1,2, \ldots, N$,

$$
\begin{aligned}
& \frac{\partial l(\ldots)}{\partial p_{k i}^{*}(\boldsymbol{\nu})} \begin{cases}=0, & p_{k i}^{*}(\boldsymbol{\nu})>0 \\
\leq 0, & p_{k i}^{*}(\boldsymbol{\nu})=0\end{cases} \\
& \frac{\partial l(\ldots)}{\partial w_{a}^{*}(\boldsymbol{\nu})}=0 \\
& \frac{\partial l(\ldots)}{\partial w_{m i}^{*}(\boldsymbol{\nu})}\left\{\begin{array}{ll}
=0, & w_{m i}^{*}(\boldsymbol{\nu})>0 \\
\leq 0, & w_{m i}^{*}(\boldsymbol{\nu})=0
\end{array} \quad \text {, for } m=1,3\right. \\
& \lambda^{*}\left(E \left[\sum_{i=1}^{N} w_{1 i}^{*}(\boldsymbol{\nu}) p_{1 i}^{*}(\boldsymbol{\nu})+\left(w_{a}^{*}(\boldsymbol{\nu})-w_{1 i}^{*}(\boldsymbol{\nu})\right) p_{2 i}^{*}(\boldsymbol{\nu})\right.\right. \\
& \left.\left.+w_{3 i}^{*}(\boldsymbol{\nu}) p_{3 i}^{*}(\boldsymbol{\nu})+\left(1-w_{a}^{*}(\boldsymbol{\nu})-w_{3 i}^{*}(\boldsymbol{\nu})\right) p_{4 i}^{*}(\boldsymbol{\nu})\right]-P_{a v}\right)=0 \\
& s_{a}^{*}\left(E\left[w_{a}^{*}(\boldsymbol{\nu})\right]-\left(1-\epsilon_{s}\right)\right)=0 \\
& s_{i}^{*}\left(E\left[w_{1 i}^{*}(\boldsymbol{\nu})+w_{3 i}^{*}(\boldsymbol{\nu})\right]-\left(1-\epsilon_{p i}\right)\right)=0 \\
& q^{*}(\boldsymbol{\nu})\left(\sum_{i=1}^{N} r_{s i}\left(\boldsymbol{\nu}, \frac{w_{1 i}^{*}(\boldsymbol{\nu})}{w_{a}^{*}(\boldsymbol{\nu})} p_{1 i}^{*}(\boldsymbol{\nu})+\frac{\left(w_{a}^{*}(\boldsymbol{\nu})-w_{1 i}^{*}(\boldsymbol{\nu})\right)}{w_{a}^{*}(\boldsymbol{\nu})} p_{2 i}^{*}(\boldsymbol{\nu})\right)\right. \\
& \left.-r_{s}^{0}\right)=0 \\
& u_{i}^{*}(\boldsymbol{\nu})\left(w_{1 i}^{*}(\boldsymbol{\nu})\left[p_{r p, i}^{*}(\boldsymbol{\nu})-p_{1 i}^{*}(\boldsymbol{\nu})\right]+\right. \\
& \left.w_{3 i}^{*}(\boldsymbol{\nu})\left[p_{r p, i}^{*}(\boldsymbol{\nu})-p_{3 i}^{*}(\boldsymbol{\nu})\right]\right)=0 \\
& \gamma_{i}^{*}(\boldsymbol{\nu})\left[w_{a}^{*}(\boldsymbol{\nu})-w_{1 i}^{*}(\boldsymbol{\nu})\right]=0 \\
& \eta_{i}^{*}(\boldsymbol{\nu})\left[1-w_{a}^{*}(\boldsymbol{\nu})-w_{3 i}^{*}(\boldsymbol{\nu})\right]=0, \\
& w_{1 i}^{*}(\boldsymbol{\nu}), w_{3 i}^{*}(\boldsymbol{\nu}) \geq 0 \\
& p_{k i}^{*}(\boldsymbol{\nu}) \geq 0, k=1,2,3,4
\end{aligned}
$$




$$
\begin{aligned}
& \max _{p_{k i} \geq 0, w_{a}, w_{1 i}, w_{3 i}} E\left[\sum_{i=1}^{N} w_{1 i}(\boldsymbol{\nu}) r_{s i}\left(\boldsymbol{\nu}, p_{1 i}(\boldsymbol{\nu})\right)+\left(w_{a}(\boldsymbol{\nu})-w_{1 i}(\boldsymbol{\nu})\right) r_{s i}\left(\boldsymbol{\nu}, p_{2 i}(\boldsymbol{\nu})\right)\right. \\
& \left.+w_{3 i}(\boldsymbol{\nu}) r_{s i}\left(\boldsymbol{\nu}, p_{3 i}(\boldsymbol{\nu})\right)+\left(1-w_{a}(\boldsymbol{\nu})-w_{3 i}(\boldsymbol{\nu})\right) r_{s i}\left(\boldsymbol{\nu}, p_{4 i}(\boldsymbol{\nu})\right)\right] \\
& \text { s.t. (a) } E\left[\sum_{i=1}^{N} w_{1 i}(\boldsymbol{\nu}) p_{1 i}(\boldsymbol{\nu})+\left(w_{a}(\boldsymbol{\nu})-w_{1 i}(\boldsymbol{\nu})\right) p_{2 i}(\boldsymbol{\nu})\right. \\
& \left.+w_{3 i}(\boldsymbol{\nu}) p_{3 i}(\boldsymbol{\nu})+\left(1-w_{a}(\boldsymbol{\nu})-w_{3 i}(\boldsymbol{\nu})\right) p_{4 i}(\boldsymbol{\nu})\right] \leq P_{a v} \\
& \text { (b) } E\left[w_{a}(\boldsymbol{\nu})\right] \geq 1-\epsilon_{s}, \quad \text { (c) } E\left[w_{1 i}(\boldsymbol{\nu})+w_{3 i}(\boldsymbol{\nu})\right] \geq 1-\epsilon_{p i}, \forall i \\
& \text { (d) }\left[\sum_{i=1}^{N} r_{s i}\left(\boldsymbol{\nu}, \frac{w_{1 i}(\boldsymbol{\nu})}{w_{a}(\boldsymbol{\nu})} p_{1 i}(\boldsymbol{\nu})+\frac{\left(w_{a}(\boldsymbol{\nu})-w_{1 i}(\boldsymbol{\nu})\right)}{w_{a}(\boldsymbol{\nu})} p_{2 i}(\boldsymbol{\nu})\right)-r_{s}^{0}\right] \geq 0, \forall i \\
& \text { (e) } w_{1 i}(\boldsymbol{\nu})\left[\frac{1}{\beta_{i}}\left(\frac{g_{i} P_{p i}\left(g_{i}\right)}{e^{r_{p i}^{0}}-1}-N_{0}\right)^{+}-p_{1 i}(\boldsymbol{\nu})\right]+w_{3 i}(\boldsymbol{\nu})\left[\frac{1}{\beta_{i}}\left(\frac{g_{i} P_{p i}\left(g_{i}\right)}{e^{r_{p i}^{0}}-1}-N_{0}\right)^{+}-p_{3 i}(\boldsymbol{\nu})\right] \geq 0, \forall i
\end{aligned}
$$

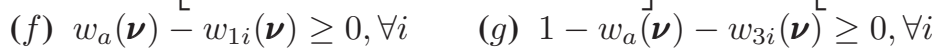

$$
\begin{aligned}
& \text { (h) } w_{1 i}(\boldsymbol{\nu}), w_{3 i}(\boldsymbol{\nu}) \geq 0, \forall i \text {. }
\end{aligned}
$$

$$
\begin{aligned}
l\left(\boldsymbol{\nu}, p_{k i}(\boldsymbol{\nu}), w_{a}(\boldsymbol{\nu}), w_{1 i}(\boldsymbol{\nu}), w_{3 i}(\boldsymbol{\nu}), \lambda, s_{a}, \mathbf{s}, q(\boldsymbol{\nu}), u_{i}(\boldsymbol{\nu}), \gamma_{i}(\boldsymbol{\nu}), \eta_{i}(\boldsymbol{\nu})\right) \\
=w_{a}(\boldsymbol{\nu})\left[s_{a}+\sum_{i=1}^{N}\left(r_{s i}\left(\boldsymbol{\nu}, p_{2 i}(\boldsymbol{\nu})\right)-\lambda p_{2 i}(\boldsymbol{\nu})+\gamma_{i}(\boldsymbol{\nu})\right)-\sum_{i=1}^{N}\left(r_{s i}\left(\boldsymbol{\nu}, p_{4 i}(\boldsymbol{\nu})\right)-\lambda p_{4 i}(\boldsymbol{\nu})+\eta_{i}(\boldsymbol{\nu})\right)\right]+ \\
\quad \sum_{i=1}^{N} w_{1 i}(\boldsymbol{\nu})\left(s_{i}+r_{s i}\left(\boldsymbol{\nu}, p_{1 i}(\boldsymbol{\nu})\right)-\lambda p_{1 i}(\boldsymbol{\nu})-\left(r_{s i}\left(\boldsymbol{\nu}, p_{2 i}(\boldsymbol{\nu})\right)-\lambda p_{2 i}(\boldsymbol{\nu})\right)-\gamma_{i}(\boldsymbol{\nu})\right)+ \\
\quad \sum_{i=1}^{N} w_{3 i}(\boldsymbol{\nu})\left(s_{i}+r_{s i}\left(\boldsymbol{\nu}, p_{3 i}(\boldsymbol{\nu})\right)-\lambda p_{3 i}(\boldsymbol{\nu})-\left(r_{s i}\left(\boldsymbol{\nu}, p_{4 i}(\boldsymbol{\nu})\right)-\lambda p_{4 i}(\boldsymbol{\nu})\right)-\eta_{i}(\boldsymbol{\nu})\right)+ \\
q(\boldsymbol{\nu})\left(\sum_{i=1}^{N} r_{s i}\left(\boldsymbol{\nu}, \frac{w_{1 i}(\boldsymbol{\nu})}{w_{a}(\boldsymbol{\nu})} p_{1 i}(\boldsymbol{\nu})+\frac{w_{a}(\boldsymbol{\nu})-w_{1 i}(\boldsymbol{\nu})}{w_{a}(\boldsymbol{\nu})} p_{2 i}(\boldsymbol{\nu})\right)-r_{s}^{0}\right)+ \\
\quad \sum_{i=1}^{N} u_{i}(\boldsymbol{\nu})\left(w_{1 i}(\boldsymbol{\nu})\left[p_{r p, i}(\boldsymbol{\nu})-p_{1 i}(\boldsymbol{\nu})\right]+w_{3 i}(\boldsymbol{\nu})\left[p_{r p, i}(\boldsymbol{\nu})-p_{3 i}(\boldsymbol{\nu})\right]\right)
\end{aligned}
$$

Using the conditions from (7) to (18), the Lagrangian at the optimal solution for each state $\nu$ must satisfy

$$
\begin{aligned}
& l(\ldots) \\
&=w_{a}^{*}(\boldsymbol{\nu})\left(\sum_{i=1}^{N}\left[r_{s i}\left(\boldsymbol{\nu}, p_{2 i}^{*}(\boldsymbol{\nu})\right)-\lambda^{*} p_{2 i}^{*}(\boldsymbol{\nu})\right]\right) \\
&-w_{a}^{*}(\boldsymbol{\nu})\left(\sum_{i=1}^{N}\left[r_{s i}\left(\boldsymbol{\nu}, p_{4 i}^{*}(\boldsymbol{\nu})\right)-\lambda^{*} p_{4 i}^{*}(\boldsymbol{\nu})\right]\right) \\
&+\sum_{i=1}^{N} w_{1 i}^{*}(\boldsymbol{\nu})\left(r_{s i}\left(\boldsymbol{\nu}, p_{1 i}^{*}(\boldsymbol{\nu})\right)-\lambda^{*} p_{1 i}^{*}(\boldsymbol{\nu})\right) \\
&-\sum_{i=1}^{N} w_{1 i}^{*}(\boldsymbol{\nu})\left(r_{s i}\left(\boldsymbol{\nu}, p_{2 i}^{*}(\boldsymbol{\nu})\right)-\lambda^{*} p_{2 i}^{*}(\boldsymbol{\nu})\right) \\
&+\sum_{i=1}^{N} w_{3 i}^{*}(\boldsymbol{\nu})\left(r_{s i}\left(\boldsymbol{\nu}, p_{3 i}^{*}(\boldsymbol{\nu})\right)-\lambda^{*} p_{3 i}^{*}(\boldsymbol{\nu})\right) \\
& \quad-\sum_{i=1}^{N} w_{3 i}^{*}(\boldsymbol{\nu})\left(r_{s i}\left(\boldsymbol{\nu}, p_{4 i}^{*}(\boldsymbol{\nu})\right)-\lambda^{*} p_{4 i}^{*}(\boldsymbol{\nu})\right) \\
&+s_{a}^{*} w_{a}^{*}(\boldsymbol{\nu})+\sum_{i=1}^{N} s_{i}^{*}\left[w_{1 i}^{*}(\boldsymbol{\nu})+w_{3 i}^{*}(\boldsymbol{\nu})\right]
\end{aligned}
$$

We now define the "subchannel benefit functions" as follows Also, define $\mathbf{p}_{w f}\left(\boldsymbol{\nu}, \lambda^{*}\right)=\left[p_{w f, 1}\left(\boldsymbol{\nu}, \lambda^{*}\right), \ldots, p_{w f, N}\left(\boldsymbol{\nu}, \lambda^{*}\right)\right]$ where $p_{w f, i}\left(\boldsymbol{\nu}, \lambda^{*}\right)=\left(\frac{1}{\lambda^{*}}-\frac{\alpha_{i} P_{p i}\left(g_{i}\right)+N_{0}}{h_{i}}\right)^{+}$. With the above definitions and notations, Theorem 1 below summarizes the optimal solution to Problem (1). Due to space constraints, a sketch of its proof is provided in the Appendix C, while the detailed proof can be found in [22].

Theorem 1: There exists a minimum average power $P_{\min }$ such that if $P_{a v}=P_{\min }$, we have $P_{s i}^{*}(\boldsymbol{\nu})=\left(X_{w_{1 i}^{*}}(\boldsymbol{\nu})+\right.$ $\left.X_{w_{2 i}^{*}}(\boldsymbol{\nu})\right) p_{r s, i}(\boldsymbol{\nu})$. If $P_{a v}>P_{\min }$, the optimal solution $P_{a v}^{*}$ is defined in (21) and the optimal solutions of $w_{a}^{*}$ and $w_{k i}^{*}$ are defined in (22). Note that $\mathscr{S}_{k}$ is the set of subchannel $i$ power policy $p_{k i}^{*} 0<\kappa_{1 i}^{*}(\boldsymbol{\nu})<w_{a}^{*}(\boldsymbol{\nu}), 0<\kappa_{3 i}^{*}(\boldsymbol{\nu})<1-w_{a}^{*}(\boldsymbol{\nu})$, and $0<\kappa_{a}^{*}(\boldsymbol{\nu})<1 . \lambda^{*}, s_{a}^{*}$, and $s_{i}^{*}, \kappa_{1 i}^{*}(\boldsymbol{\nu}), \kappa_{3 i}^{*}(\boldsymbol{\nu})$ and $\kappa_{a}^{*}(\boldsymbol{\nu})$ are the solutions to $E\left[\sum_{i=1}^{N} P_{s i}^{*}(\boldsymbol{\nu})\right]=P_{a v}, E\left[w_{a}^{*}(\boldsymbol{\nu})\right] \geq 1-\epsilon_{s}$, and $E\left[w_{1 i}^{*}(\boldsymbol{\nu})+w_{3 i}^{*}(\boldsymbol{\nu})\right] \geq 1-\epsilon_{p i}$ for all $i$, respectively.

\section{Discussions on the optimal power control scheme:}

- $P_{\min }$ denotes the minimum average power required by the SU to make Problem (1) feasible. A discussion on the feasibility condition will be provided in Section IV.A.

- For continuous fading channels, $\mathscr{S}_{1} \cap \mathscr{S}_{2}$ and $\mathscr{S}_{3} \cap \mathscr{S}_{4}$ are empty sets since $\mathscr{B}_{1 i, \boldsymbol{\nu}}=\mathscr{B}_{2 i, \boldsymbol{\nu}}$ and $\mathscr{B}_{3 i, \boldsymbol{\nu}}=\mathscr{B}_{4 i, \boldsymbol{\nu}}$ can occur with probability of measure zero for every $i$, making the optimal power allocation scheme deterministic, i.e. only one deterministic power strategy is used for each $\nu$. Obviously, there are at most $2^{N}+1$ possible candidate power control policies, i.e. each channel can use $p_{w f, i}$ or $p_{r p, i}$ or all channels use $\mathbf{p}_{r s}$. When $N=1$, this reduces to only 3 possible power policies, as presented in [15]. This raises the question regarding the necessity of using a probabilistic power allocation policy for continuous fading channels. The justification behind using a probabilistic power allocation law as a candidate for an optimal policy is twofold. First and foremost this transforms Problem (1) to a convex optimization problem (5) which can then easily be solved using the necessary and sufficient KKT conditions. In addition, this makes the optimal power allocation law applicable to general fading distributions which may have discrete components. In the case of continuous fading distributions (as considered in the Numerical results section in this paper), the probabilistic power allocation law simplifies to a switching 


$$
\begin{array}{lll}
\mathscr{B}_{1 i}\left(\boldsymbol{\nu}, p_{1 i}^{*}(\boldsymbol{\nu}), \lambda^{*}, s_{i}^{*}\right) & \triangleq \mathscr{B}_{1 i, \boldsymbol{\nu}} \triangleq r_{s i}\left(\boldsymbol{\nu}, p_{1 i}^{*}(\boldsymbol{\nu})\right)-\lambda^{*} p_{1 i}^{*}(\boldsymbol{\nu})+s_{i}^{*} \\
\mathscr{B}_{2 i}\left(\boldsymbol{\nu}, p_{2 i}^{*}(\boldsymbol{\nu}), \lambda^{*}\right) & \triangleq \mathscr{B}_{2 i, \boldsymbol{\nu}} \triangleq r_{s i}\left(\boldsymbol{\nu}, p_{2 i}^{*}(\boldsymbol{\nu})\right)-\lambda^{*} p_{2 i}^{*}(\boldsymbol{\nu}) \\
\mathscr{B}_{3 i}\left(\boldsymbol{\nu}, p_{3 i}^{*}(\boldsymbol{\nu}), \lambda^{*}, s_{i}^{*}\right) & \triangleq \mathscr{B}_{3 i, \boldsymbol{\nu}} \triangleq r_{s i}\left(\boldsymbol{\nu}, p_{3 i}^{*}(\boldsymbol{\nu})\right)-\lambda^{*} p_{3 i}^{*}(\boldsymbol{\nu})+s_{i}^{*} \\
\mathscr{B}_{4 i}\left(\boldsymbol{\nu}, p_{4 i}^{*}(\boldsymbol{\nu}), \lambda^{*}\right) & \triangleq \mathscr{B}_{4 i, \boldsymbol{\nu}} \triangleq r_{s i}\left(\boldsymbol{\nu}, p_{4 i}^{*}(\boldsymbol{\nu})\right)-\lambda^{*} p_{4 i}^{*}(\boldsymbol{\nu})
\end{array}
$$

$$
\begin{aligned}
& P_{s i}^{*}(\boldsymbol{\nu}) \\
& =p_{w f, i}\left(\boldsymbol{\nu}, \lambda^{*}\right)+X_{w_{3 i}^{*}}(\boldsymbol{\nu})\left[-\left(p_{w f, i}\left(\boldsymbol{\nu}, \lambda^{*}\right)-p_{r p, i}(\boldsymbol{\nu})\right)^{+}\right] \\
& +X_{w_{2 i}^{*}}(\boldsymbol{\nu})\left[\left(p_{r s, i}(\boldsymbol{\nu})-p_{w f, i}\left(\boldsymbol{\nu}, \lambda^{*}\right)\right)^{+}\right] \\
& +X_{w_{1 i}^{*}}(\boldsymbol{\nu})\left[\left(p_{r s, i}(\boldsymbol{\nu})-p_{w f, i}\left(\boldsymbol{\nu}, \lambda^{*}\right)\right)^{+}-\left(p_{w f, i}\left(\boldsymbol{\nu}, \lambda^{*}\right)-p_{r p, i}(\boldsymbol{\nu})\right)^{+}\right]
\end{aligned}
$$

$$
\begin{aligned}
& w_{1 i}^{*}(\boldsymbol{\nu})=\left\{\begin{array}{ll}
0, & \mathscr{B}_{1 i, \boldsymbol{\nu}} \leq \mathscr{B}_{2 i, \boldsymbol{\nu}} \\
\kappa_{1 i}^{*}(\boldsymbol{\nu}), & \mathscr{B}_{1 i, \boldsymbol{\nu}}=\mathscr{B}_{2 i, \boldsymbol{\nu}} \\
w_{a}^{*}(\boldsymbol{\nu}), & \mathscr{B}_{1 i, \boldsymbol{\nu}} \geq \mathscr{B}_{2 i, \boldsymbol{\nu}}
\end{array},\right. \\
& w_{2 i}^{*}(\boldsymbol{\nu})=w_{a}^{*}(\boldsymbol{\nu})-w_{1 i}^{*}(\boldsymbol{\nu}) \\
& w_{3 i}^{*}(\boldsymbol{\nu})= \begin{cases}0, & \mathscr{B}_{3 i, \boldsymbol{\nu}} \leq \mathscr{B}_{4 i, \boldsymbol{\nu}} \\
\kappa_{3 i}^{*}(\boldsymbol{\nu}), & \mathscr{B}_{3 i, \boldsymbol{\nu}}=\mathscr{B}_{4 i, \boldsymbol{\nu}} \\
1-w_{a}^{*}(\boldsymbol{\nu}), & \mathscr{B}_{3 i, \boldsymbol{\nu}} \geq \mathscr{B}_{4 i, \boldsymbol{\nu}}\end{cases} \\
& w_{4 i}^{*}(\boldsymbol{\nu})=\left(1-w_{a}^{*}(\boldsymbol{\nu})\right)-w_{3 i}^{*}(\boldsymbol{\nu}), \\
& w_{a}^{*}(\boldsymbol{\nu})= \begin{cases}0, \quad & s_{a}^{*}+\sum_{i \in \mathscr{S}_{1}} \mathscr{B}_{1 i, \boldsymbol{\nu}}+\sum_{i \in \mathscr{S}_{2}} \mathscr{B}_{2 i, \boldsymbol{\nu}}-\sum_{i \in \mathscr{S}_{1} \cap \mathscr{S}_{2}} \mathscr{B}_{1 i, \boldsymbol{\nu}} \\
& \leq \sum_{i \in \mathscr{S}_{3}} \mathscr{B}_{3 i, \boldsymbol{\nu}}+\sum_{i \in \mathscr{S}_{4}} \mathscr{B}_{4 i, \boldsymbol{\nu}}-\sum_{i \in \mathscr{S}_{3} \cap \mathscr{S}_{4}} \mathscr{B}_{3 i, \boldsymbol{\nu}} \\
\kappa_{a}^{*}(\boldsymbol{\nu}), & s_{a}^{*}+\sum_{i \in \mathscr{S}_{1}} \mathscr{B}_{1 i, \boldsymbol{\nu}}+\sum_{i \in \mathscr{S}_{2}} \mathscr{B}_{2 i, \boldsymbol{\nu}}-\sum_{i \in \mathscr{S}_{1} \cap \mathscr{S}_{2}} \mathscr{B}_{1 i, \boldsymbol{\nu}} \\
& =\sum_{i \in \mathscr{S}_{3}} \mathscr{B}_{3 i, \boldsymbol{\nu}}+\sum_{i \in \mathscr{S}_{4}} \mathscr{B}_{4 i, \boldsymbol{\nu}}-\sum_{i \in \mathscr{S}_{3} \cap \mathscr{S}_{4}} \mathscr{B}_{3 i, \boldsymbol{\nu}} \\
& s_{a}^{*}+\sum_{i \in \mathscr{S}_{1}} \mathscr{B}_{1 i, \boldsymbol{\nu}}+\sum_{i \in \mathscr{S}_{2}} \mathscr{B}_{2 i, \boldsymbol{\nu}}-\sum_{i \in \mathscr{S}_{1} \cap \mathscr{S}_{2}} \mathscr{B}_{1 i, \boldsymbol{\nu}} \\
& \geq \sum_{i \in \mathscr{S}_{3}} \mathscr{B}_{3 i, \boldsymbol{\nu}}+\sum_{i \in \mathscr{S}_{4}} \mathscr{B}_{4 i, \boldsymbol{\nu}}-\sum_{i \in \mathscr{S}_{3} \cap \mathscr{S}_{4}} \mathscr{B}_{3 i, \boldsymbol{\nu}}\end{cases}
\end{aligned}
$$

policy amongst a number of deterministic policies (as explained above). In this case, the benefit of using a probabilistic power allocation technique lies in making the original problem convex and thus easily solvable. More details on this can be found in [14] for the noncognitive setting.

In order to compute the optimal power allocation policy, we first compute $\mathbf{p}_{r s}(\boldsymbol{\nu})$ and $\mathbf{p}_{w f}\left(\boldsymbol{\nu}, \lambda^{*}\right)$ and $p_{r p, i}(\boldsymbol{\nu})$ for every subchannel for a given $\nu$. Then, the possibility of using each of these deterministic schemes is checked, e.g. $\mathbf{p}_{r s}(\boldsymbol{\nu})$ can be used only when $\mathbf{p}_{w f}\left(\boldsymbol{\nu}, \lambda^{*}\right) \prec \mathbf{p}_{r s}(\boldsymbol{\nu})$ for all subchannels and, for the $i$-th subchannel, $p_{r p, i}(\boldsymbol{\nu})$ can be used only when $p_{r p, i}(\boldsymbol{\nu})>p_{w f, i}\left(\boldsymbol{\nu}, \lambda^{*}\right)$. From the remaining deterministic power allocation policies, for a given channel state, SU selects the power policy that results in the largest value of the Lagrangian function (19), which can be interpreted as the total benefit for the SU. Essentially, for a price of $\lambda^{*} \sum_{i=1}^{N} P_{s i}^{*}(\boldsymbol{\nu})$, the SU gets a rate $r_{s}\left(\boldsymbol{\nu}, \boldsymbol{P}_{s}^{*}(\boldsymbol{\nu})\right)$ and gains a benefit of $s_{a}^{*}$ if $\mathrm{SU}$ is not in outage and gains a benefit of $s_{i}^{*}$ if the $i$-th PU is not in outage. Note that these power allocation policies are computed (for every channel state) as a function of a fixed set of values of the Lagrange multipliers. In order to determine the optimal values of the Lagrange multipliers, one can use either subgradient based iterative methods or other appropriate numerical search methods in order to satisfy the average SU transmit power and the SU and PU outage probability constraints.

Note that for every channel realization, one has to find an optimal policy from a set of $2^{N}+1$ policies, which can be computationally prohibitive to implement in real time if $N$ is large. Thus motivated, we propose a suboptimal power scheme in Section IV.B which reduces the number of candidate power allocation policies to just three for each channel realization.

- Additionally, we emphasize the role of $\lambda^{*}$ in the derived optimal power allocation scheme. When $\lambda^{*}$ is large, implying that the SU has a low power budget $P_{a v}$, SU is more likely to adopt the policy $\boldsymbol{P}_{s}^{*}(\boldsymbol{\nu})=\mathbf{p}_{r s}(\boldsymbol{\nu})$ to support its target rate. However, if $\lambda^{*}$ is small corresponding to a high SU $P_{a v}$ budget, it tends to use $p_{r p, i}(\boldsymbol{\nu})$ in each subchannel to avoid causing disruptions to $\mathrm{PU}_{i}$ 's communication. Also, if all POCs and SOC are dropped, $\lambda^{*}$ simply controls the threshold corresponding to the well known water-filling power allocation policy represented by $\mathbf{p}_{w f}(\boldsymbol{\nu})$. 


\section{A. Feasibility of the service-outage problem}

It can be seen from Theorem 1 that an average power budget $P_{a v} \geq P_{\min }$ is required for Problem (1) to be feasible, i.e. the $\mathrm{SU}$ requires a minimum average power $P_{\min }$ to at least support the service rate while satisfying all the POCs. The feasibility of the problem (1) is therefore directly related to the problem of SU outage capacity maximization subject to all $N$ POCs and an average SU transmission power constraint. In order to compute $P_{\min }$, it is therefore necessary to address the optimal power control problem for SU's outage probability (SO) minimization problem subject to all POCs and ATPC..

The SO minimization problem subject to $N$ POCs and SU's ATPC can be expressed as follows:

$$
\begin{array}{cl}
\min _{\mathbf{P}_{s}(\boldsymbol{\nu}) \succeq 0} & \operatorname{Pr}\left\{\sum_{i=1}^{N} r_{s i}\left(\boldsymbol{\nu}, P_{s i}(\boldsymbol{\nu})\right)<r_{s}^{0}\right\} \\
\text { s.t. } & \text { (a) } E\left[\sum_{i=1}^{N} P_{s i}(\boldsymbol{\nu})\right] \leq P_{a v}, \\
& \text { (b) } \operatorname{Pr}\left\{r_{p i}\left(\boldsymbol{\nu}, P_{s i}(\boldsymbol{\nu})\right)<r_{p i}^{0}\right\} \leq \epsilon_{p i}, \forall i
\end{array}
$$

Note that Problem (23) can be regarded as an extension of [11] to the $N>1$ case. This problem can also be solved using the probabilistic power allocation technique. By using the definition of $w_{k i}^{*}(\boldsymbol{\nu})$ and $p_{k i}^{*}(\boldsymbol{\nu})$ as shown in (3) and (4), we can prove the following result shown in Theorem 2 which presents the optimal power allocation policy for Problem (23). The proof is similar to that of Theorem 1 and is therefore omitted to avoid repetition.

Theorem 2: The optimal solution for $(23)$ is $P_{s i}^{*}(\boldsymbol{\nu})=$ $\left(X_{w_{1 i}^{*}}(\boldsymbol{\nu})+X_{w_{2 i}^{*}}(\boldsymbol{\nu})\right) p_{r s, i}(\boldsymbol{\nu})=X_{w_{a}^{*}}(\boldsymbol{\nu}) p_{r s, i}(\boldsymbol{\nu})$, where

$$
\begin{aligned}
& w_{1 i}^{*}(\boldsymbol{\nu})=\left\{\begin{array}{ll}
w_{a}^{*}(\boldsymbol{\nu}) & , i \in \mathscr{M} \\
0 & , i \notin \mathscr{M}
\end{array},\right. \\
& w_{2 i}^{*}(\boldsymbol{\nu})=w_{a}^{*}(\boldsymbol{\nu})-w_{1 i}^{*}(\boldsymbol{\nu}) \\
& w_{3 i}^{*}(\boldsymbol{\nu})=\left\{\begin{array}{ll}
1-w_{a}^{*}(\boldsymbol{\nu}) & , i \in \mathscr{J} \\
0 & , i \notin \mathscr{J}
\end{array},\right. \\
& w_{4 i}^{*}(\boldsymbol{\nu})=\left(1-w_{a}^{*}(\boldsymbol{\nu})\right)-w_{3 i}^{*}(\boldsymbol{\nu}), \\
& w_{a}^{*}(\boldsymbol{\nu})= \begin{cases}1 & , \sum_{i \in \mathscr{J}} S_{i}^{*} \leq 1-\Lambda^{*} \sum_{i=1}^{N} p_{r s, i}(\boldsymbol{\nu})+\sum_{i \in \mathscr{M}} S_{i}^{*} \\
\kappa_{a o}^{*}(\boldsymbol{\nu}) & , \sum_{i \in \mathscr{J}} S_{i}^{*}=1-\Lambda^{*} \sum_{i=1}^{N} p_{r s, i}(\boldsymbol{\nu})+\sum_{i \in \mathscr{M}} S_{i}^{*} \\
0 & , \sum_{i \in \mathscr{J}} S_{i}^{*} \geq 1-\Lambda^{*} \sum_{i=1}^{N} p_{r s, i}(\boldsymbol{\nu})+\sum_{i \in \mathscr{M}} S_{i}^{*}\end{cases}
\end{aligned}
$$

where $\mathscr{J}=\left\{j \mid r_{p j}(\boldsymbol{\nu}, 0) \geq r_{p j}^{0}\right\}$ and $\mathscr{M}=$ $\left\{m \mid r_{p m}\left(\boldsymbol{\nu}, p_{r s, m}\right) \geq r_{p m}^{0}\right\}$, and $\mathscr{M} \subseteq \mathscr{J} . \Lambda^{*}, S_{i}^{*}$ and $\kappa_{a o}^{*}(\boldsymbol{\nu})$ are solutions to the constraints $E\left[\sum_{i=1}^{N} P_{s i}^{*}(\boldsymbol{\nu})\right]=P_{a v}$ and $E\left[w_{1 i}^{*}(\boldsymbol{\nu})+w_{3 i}^{*}(\boldsymbol{\nu})\right] \geq 1-\epsilon_{p i}$ for all $i$.

Table I reveals the possible non-negative weighting functions $w_{k i}(\boldsymbol{\nu})$ in each subchannel and corresponding power control policies $p_{k i}(\boldsymbol{\nu})$ for a given channel state $\boldsymbol{\nu}$, leading to the final result in Theorem 2 which shows that there are 2 deterministic power schemes involved in this problem. Either the SU is OFF in order to allow an outage or it is ON with power policy $\mathbf{p}_{r s}^{*}(\boldsymbol{\nu})$. In other words, the SU decides to gain a benefit of $\sum_{i \in \mathscr{J}} S_{i}^{*}$ when it keeps silent and a benefit of
$1-\Lambda^{*} \sum_{i=1}^{N} p_{r s, i}(\boldsymbol{\nu})+\sum_{i \in \mathscr{M}} S_{i}^{*}$ when it is active. It is to be noted also that $S_{i}^{*}$ represents the benefit when $\mathrm{PU}_{i}$ is not in outage. It is not hard to show that the solution above can be specialized to the one proposed in [11] when $N=1$ and can also be specialized to the solution of the SO minimization problem without POCs in parallel fading channels [16].

We now go back to the discussion on feasibility of Problem (23) and how to compute $P_{\min }$. Clearly, it is directly related to SU's $\epsilon_{s}$-outage capacity which can be computed through the solution to Problem (23). Let $C_{\epsilon_{s}}\left(P_{a v}, \mathbf{r}_{p}^{0}, \boldsymbol{\epsilon}_{p}\right)$ stand for SU's $\epsilon_{s}$-outage capacity with POCs given fixed $\mathbf{r}_{p}^{0}, \boldsymbol{\epsilon}_{p}, \epsilon_{s}$, and $P_{a v} . C_{\epsilon_{s}}\left(P_{a v}, \mathbf{r}_{p}^{0}, \boldsymbol{\epsilon}_{p}\right)$ is the maximum instantaneous rate at which the SU can transmit with an outage probability $\epsilon_{s}$ under POCs. So, problem (1) is feasible iff the SU target rate $r_{s}^{0} \leq C_{\epsilon_{s}}\left(P_{a v}, \mathbf{r}_{p}^{0}, \boldsymbol{\epsilon}_{p}\right)$. For simplicity, let $P_{\min }\left(r_{s}^{0}, \mathbf{r}_{p}^{0}, \boldsymbol{\epsilon}_{p}\right)=$ $P_{\min }$ be the minimum SU average power needed to support $r_{s}^{0}$ with outage probability $\epsilon_{s}$ while satisfying all $N$ POCs. Hence, the feasibility condition for our main problem is then $P_{a v} \geq P_{\min }$. If $P_{a v}=P_{\min }$, then $\mathbf{p}_{1}^{*}(\boldsymbol{\nu})=\mathbf{p}_{2}^{*}(\boldsymbol{\nu})=\mathbf{p}_{r s}(\boldsymbol{\nu})$ and $\mathbf{p}_{3}^{*}(\boldsymbol{\nu})=\mathbf{p}_{4}^{*}(\boldsymbol{\nu})=0$ due to Theorem 2 .

To compute $P_{\min }$, we search for the optimal $\Lambda^{*}$ and all $S_{i}^{*}$ that solve $E\left[w_{a}^{*}(\boldsymbol{\nu})\right]=1-\epsilon_{s}, E\left[w_{1 i}^{*}(\boldsymbol{\nu})+w_{3 i}^{*}(\boldsymbol{\nu})\right] \geq 1-\epsilon_{p i}$ for all $i$. Once $\Lambda^{*}$ and $S_{i}^{*}$ are obtained, $P_{\min }$ can be computed as $E\left[\left\langle\mathbf{P}_{s}^{*}\right\rangle\right]$.

\section{B. Suboptimal power control}

The optimal solution to Problem (1) shows that the optimal power allocation policy can be evaluated to be one of at most $2^{N}+1$ candidate policies for every channel realization $\nu$, based on the pre-computed Lagrange multipliers $\lambda, s_{a}$, and all $s_{i}$, leading to a high computational complexity when $N$ is large. In this section we will investigate a suboptimal power control policy which can help lower this computational burden. Note that the Lagrange multipliers $\lambda, s_{a}$, and all $s_{i}$ can be computed off-line as they depend on long term average constraints, and thus only on the channel statistics and not on the instantaneous channel values. Below we show that our suboptimal power allocation scheme reduces the number of candidate power control policies from at most $2^{N}+1$ candidate policies to only at most 3 candidate policies.

We first make some useful observations about the optimal power allocation solution presented in Theorem 1.

- If $s_{a}^{*}=0$, the optimization problem reduces to $N$ separate optimization subproblems coupled by the same $\lambda^{*}$, allowing each subchannel of SU to select a power strategy between $p_{w f, i}(\boldsymbol{\nu})$ and $p_{w f, i}(\boldsymbol{\nu})-\left(p_{w f, i}(\boldsymbol{\nu})-p_{r p, i}(\boldsymbol{\nu})\right)^{+}$ independently of the strategies used in other subchannels. Roughly speaking, the SU is always allowed to transmit with $p_{w f, i}$ when $p_{w f, i}<p_{r p, i}$. Otherwise, SU has to decide whether $p_{w f, i}(\boldsymbol{\nu})$ or $p_{r p, i}(\boldsymbol{\nu})$ will return a higher benefit. It is not hard to show that if there exists some $s_{i}^{*}=0$, the corresponding power control in that $i$-th subchannel is $p_{w f, i}$. Furthermore, note that there is no need to use the strategy $\mathbf{p}_{r s}(\boldsymbol{\nu})$ since the SOC is inactive.

- If $s_{a}^{*}>0$ and $s_{i}^{*}=0$ for all $i$, there are just two candidate power strategies which are $\mathbf{p}_{w f}(\boldsymbol{\nu})+\left(\mathbf{p}_{r s}(\boldsymbol{\nu})-\mathbf{p}_{w f}(\boldsymbol{\nu})\right)^{+}$ and $\mathbf{p}_{w f}(\boldsymbol{\nu})$. In this case, all POCs are inactive. 
TABLE I

THREE POSSIBLE CASES IN $i$-TH SUBCHANNEL FOR A GIVEN $\boldsymbol{\nu}$

\begin{tabular}{cll}
\hline \hline Cases & Power allocation policy & Candidate non-negative $w_{k i}$ \\
\hline$r_{p i}\left(\boldsymbol{\nu}, p_{r s, i}\right) \geq r_{p i}^{0}$ and $r_{p i}(\boldsymbol{\nu}, 0) \geq r_{p i}^{0}$ & $p_{1 i}^{*}=p_{r s, i}$ and $p_{3 i}^{*}=0$ & $w_{1 i}^{*}=w_{a}^{*}, w_{3 i}^{*}=1-w_{a}^{*}$ \\
$r_{p i}\left(\boldsymbol{\nu}, p_{r s, i}\right)<r_{p i}^{0}$ and $r_{p i}(\boldsymbol{\nu}, 0) \geq r_{p i}^{0}$ & $p_{2 i}^{*}=p_{r s, i}$ and $p_{3 i}^{*}=0$ & $w_{2 i}^{*}=1-w_{1 i}^{*}=w_{a}^{*}, w_{3 i}^{*}=1-w_{a}^{*}$ \\
$r_{p i}\left(\boldsymbol{\nu}, p_{r s, i}\right)<r_{p i}^{0}$ and $r_{p i}(\boldsymbol{\nu}, 0)<r_{p i}^{0}$ & $p_{2 i}^{*}=p_{r s, i}$ and $p_{4 i}^{*}=0$ & $w_{2 i}^{*}=1-w_{1 i}^{*}=w_{a}^{*}, w_{4 i}^{*}=1-w_{a}^{*}$ \\
\hline \hline
\end{tabular}

- The least complicated circumstance arises when both $s_{a}^{*}$ and all $s_{i}^{*}$ are zero. The optimal power control is just $\mathbf{p}_{w f}(\boldsymbol{\nu})$.

- The most complicated scenario arises when both $s_{a}^{*}$ and all $s_{i}^{*}$ are positive. In this case, the $\mathrm{SU}$ can either decide to transmit with $\mathbf{p}_{w f}(\boldsymbol{\nu})+\left(\mathbf{p}_{r s}(\boldsymbol{\nu})-\mathbf{p}_{w f}(\boldsymbol{\nu})\right)^{+}$or select whether to transmit with $p_{w f, i}(\boldsymbol{\nu})$ or with $p_{w f, i}(\boldsymbol{\nu})-$ $\left(p_{w f, i}(\boldsymbol{\nu})-p_{r p, i}(\boldsymbol{\nu})\right)^{+}$in the $i$-th band for a given channel state $\boldsymbol{\nu}$. Thus the SU has to select one strategy out of at most $2^{N}+1$ strategies for every realization $\boldsymbol{\nu}$. Moreover, the tradeoff between satisfying the SU service rate and avoiding causing PU outages makes the optimal power strategy impossible to be selected independently in each subchannel.

The proposed suboptimal power control will therefore focus on the worst case scenario when both $s_{a}^{*}$ and some or all $s_{i}^{*}$ are positive, so that the number of candidate power strategies in each $\nu$ is substantially reduced while sacrificing a small SU ergodic capacity loss. Note that for the first three cases above, the suboptimal power control is kept the same as the optimal one due to its low complexity. However, the remaining power strategies must be selected carefully since the SU must satisfy both SOC and all POCs. Below we describe how we choose the three candidate power vector policies $\mathbf{P}_{1}, \mathbf{P}_{2}$ and $\mathbf{P}_{3}$ ) which constitute the suboptimal power control policies for each $\nu$.

(1): $\mathbf{P}_{1}=\mathbf{p}_{w f}(\boldsymbol{\nu})$ is utilized when SU decides to neglect the outage situation for all PUs and SU itself.

(2): $\mathbf{P}_{2}=\mathbf{p}_{w f}(\boldsymbol{\nu})+\left(\mathbf{p}_{r s}(\boldsymbol{\nu})-\mathbf{p}_{w f}(\boldsymbol{\nu})\right)^{+}$is kept as one of the candidates since it helps $\mathrm{SU}$ support its service rate.

(3): For $\mathbf{P}_{3}$, we assign the $i$-th power element to be $P_{3 i}=$ $p_{w f, i}$ for $s_{i}^{*}=0$. For $s_{i}^{*}>0, P_{3 i}=p_{w f, i}(\boldsymbol{\nu})-\left(p_{w f, i}(\boldsymbol{\nu})-\right.$ $\left.p_{r p, i}(\boldsymbol{\nu})\right)^{+}$. Note that this power policy is used to prevent PUs' outage situation in all subchannels (1 power policy) instead of considering all possible candidates that can protect each subchannel (up to $2^{N}$ policies) in the optimal solution.

In our proposed suboptimal power control, the number of candidate power strategies reduces from at most $2^{N}+1$ to just 3 for each $\nu$. Furthermore, it is not hard to show that this suboptimal scheme is in fact optimal when $N=1$.

\section{NumericAl RESUlts}

In this section, we will illustrate via simulation results the performance of the optimal solutions to the service-outage based capacity optimization problem (1), the generalized SO minimization problem (23), and also the performance of the suboptimal power allocation policy. All channel power gains are assumed to be mutually independent and exponentially distributed (Rayleigh fading) with unit mean. Noises at all

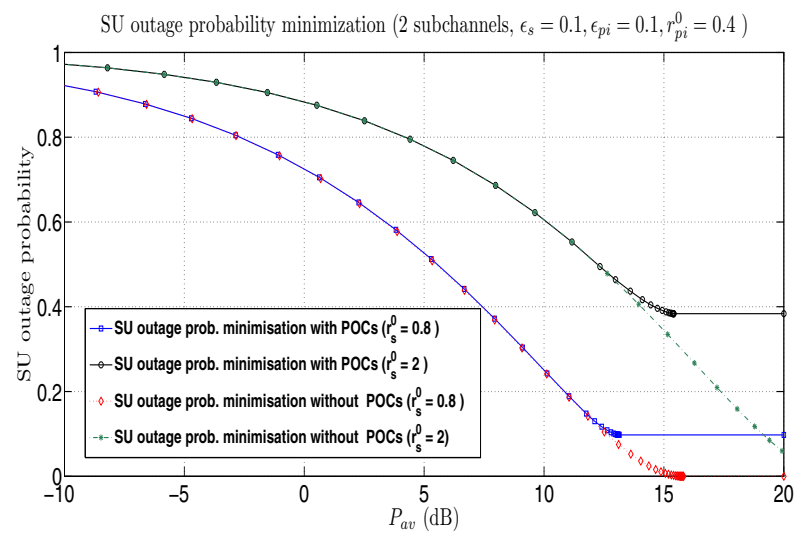

Fig. 1. SU outage probability performance from SU outage probability minimization problem with POCs with varying $r_{s}^{0}\left(\epsilon_{p i}=0.1, \epsilon_{s}=\right.$ $\left.0.1, r_{p i}^{0}=0.4\right)$

PR and SR for each subchannel are presumed to be equal and AWGN with a normalized unit variance, i.e. $N_{0}=1$. This allows us to consider the SU and PU transmit powers and the SU average transmit power constraint in unitless terms, and thus expressed in $\mathrm{dB}$. Note also that the SU average transmission power can be regarded as the average transmit SNR at the SU transmitter. We assume that all PUs are symmetric with identical transmit power $P_{c i}=15 \mathrm{~dB}$, identical maximum outage probability $\epsilon_{p i}=0.1$, and identical service rates, the exact value of which will be specified within the individual simulation descriptions below. The SU outage probability threshold $\epsilon_{s}$ is also set to be 0.1 . The units of the service rates for $\mathrm{SU}$ and $\mathrm{PU}$ are expressed in nats/transmission. In the various figures illustrating the simulation results, $R_{s}$ (in nats/transmission) denotes the SU ergodic capacity achieved under various combinations of primary and secondary outage constraints.

\section{A. SO minimization problem}

Fig. 1 illustrates the SU outage probability performance of the optimal solution to Problem (23) for $N=2$ subchannels with $r_{s}^{0}=0.8, r_{s}^{0}=2$ when $r_{p i}^{0}=0.4$. The results for the $\mathrm{SO}$ minimization problem without POCs are also included as a lower bound to the solution to Problem (23). Noticeably, POCs make the outage probability graph saturate in the high average power region, i.e. the SU cannot reduce its outage probability substantially regardless of how large its average transmission power budget is. Also as expected, the SU can have a lower outage probability when the service rate is lower. For a fixed $r_{s}^{0}=0.8$, Fig. 2 illustrates the SU outage probability performance for various values of $r_{p i}^{0}$. Clearly, SU can achieve a lower outage probability when PUs' service rates 


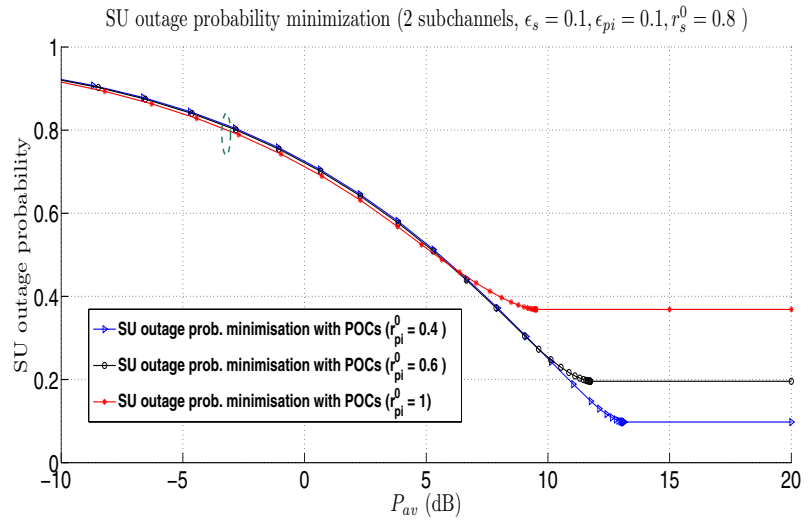

Fig. 2. SU outage probability performance from SU outage probability minimization problem with POCs with varying $r_{p i}^{0}\left(\epsilon_{p i}=0.1, \epsilon_{s}=\right.$ $\left.0.1, r_{s}^{0}=0.8\right)$

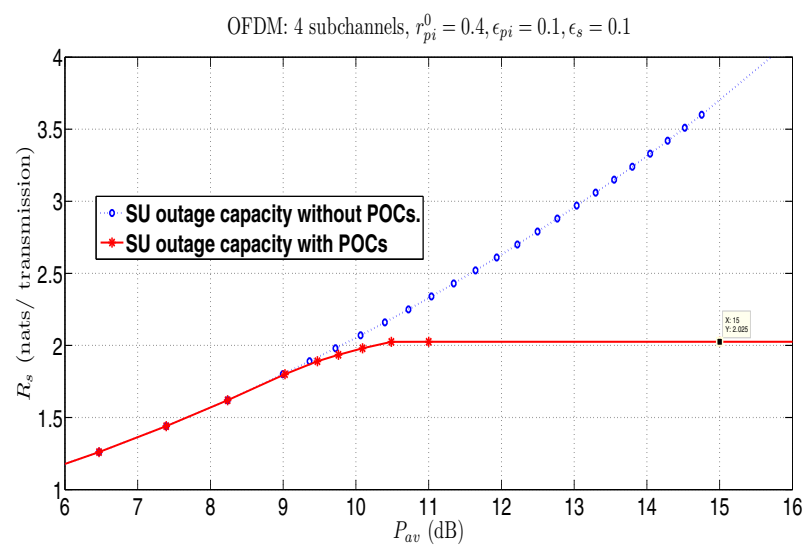

Fig. 3. SU outage capacity versus average power budget from SU outage probability minimization problem with 4 subchannels $\left(\epsilon_{p i}=0.1, \epsilon_{s}=\right.$ $0.1, r_{p i}^{0}=0.4$ )

are decreased. However, one may wonder why the SU outage probability with higher PUs' target rates are marginally better in the low SNR region (indicated by the green oval). This is due to the ON-OFF PU power control policy. In particular, in the low SNR region, with higher $r_{p i}^{0}, \mathrm{PU}_{i}$ is more likely to turn OFF and thus be in outage since the threshold of $g_{T, i}$ is high. This allows the SU more opportunities to transmit. However, at high SNR, POCs become active and they get stricter with higher $r_{p i}^{0}$, thus restricting the $\mathrm{SU}$ transmission opportunities, and increasing its outage probability. In Fig.3, we compare the SU $\epsilon_{s}$-outage capacities with POCs and without POCs for $N=4$ subchannels. Obviously, the one without POCs will serve as an upper bound of the one with POCs. The results also show that the under the POCs, the SU outage capacity eventually saturate and cannot increase any further, regardless of the average transmission power. This implies that the SU cannot have a service rate $r_{s}^{0}$ to be more than a certain 'maximum target rate', say $r_{s, M A X}^{0}$ when the POCs are present. This observation is important for Problem (1) since this problem is feasible iff $P_{a v} \geq P_{\min }$ where $P_{\min }$ is the minimum power that can support $\mathrm{SU} \epsilon_{s}$-outage capacity $r_{s}^{0}$ when the POCs are present. Hence, if $r_{s}^{0}>r_{s, M A X}^{0}$, then $P_{\min }$ becomes infinity.

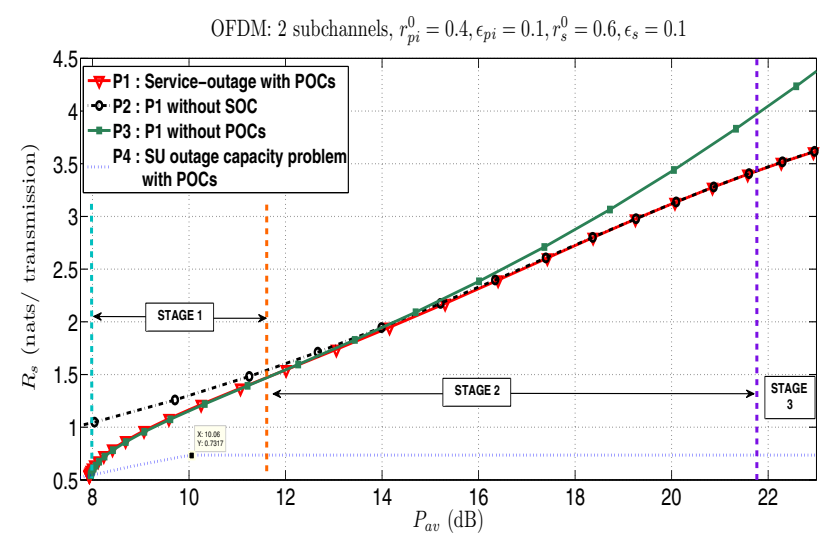

Fig. 4. Average SU rate performance from SU Ergodic capacity maximization problem with POC and SOC with related bounds $\left(\epsilon_{p i}=0.1, \epsilon_{s}=0.1, r_{p i}^{0}=\right.$ $\left.0.4, r_{s}^{0}=0.6\right)$

\section{B. SEC maximization problem}

In Fig.4, we compare the the SU ergodic capacity achieved by the optimal solution to Problem (1) with related bounds for 2 subchannels with $r_{s}^{0}=0.6$ and $r_{p i}^{0}=0.4$. The performance of the optimal solution to Problem (1) is denoted by (P1). The optimal SU ergodic capacity performance when the POCs are discarded and only SOC is present, is represented by (P3), and the SU ergodic capacity performance with the POCs present and SOC discarded is represented by (P2), whereas (P4) represents the $\mathrm{SU} \epsilon_{s}$-outage capacity with all the POCs present. As expected, (P1) and (P4) start at the same point $P_{a v}=P_{\min }$, which is the minimum power necessary for feasibility of Problem (1). As the available average power $P_{a v}$ increases, the SU ergodic capacity achieved as a solution to Problem (1) can enter up to three different stages, as shown in Fig. 4. In the first stage, the ergodic capacity graphs (P1) and (P3) are identical since POCs are still inactive. Once at least one POC becomes active (in the second stage), (P1) is upper bounded by both (P3) and (P2). Eventually, as $P_{a v}$ increases even further, (P1) becomes identical with (P2) when the SOC become completely inactive in the third stage.

However, it should be clarified that there may not always be a third stage as illustrated by Fig.5(a) where $r_{s}^{0}$ is varied while all $r_{p i}^{0}$ are still kept at 0.4 . Note that in the third stage, the SU outage constraint becomes inactive, thus, in this stage the SU ergodic capacity results should be independent of $r_{s}^{0}$. As seen in Fig.5(a), the SU ergodic capacity graphs of $r_{s}^{0}=0.4$ and $r_{s}^{0}=0.6$ become strictly positive at different $P_{a v}$ (due to different $P_{\min }$ requirements), but they become exactly the same when $P_{a v}$ is high enough which implies that they reach the third stage where SOC becomes inactive. However,this is not the case when $r_{s}^{0}$ is considerably high such that the power strategy $\mathbf{p}_{r s}$ is unavoidable for some channel states. Indeed, when $r_{s}^{0}=0.8$, the SU ergodic capacity does not enter the third stage no matter how high $P_{a v}$ is, as illustrated by Fig.5(b), via the performance gap with the SU ergodic capacities for $r_{s}^{0}=0.4$ and $r_{s}^{0}=0.6$. Additionally, in Fig. 6, the effect of a noisy estimated channel gain between each $\mathrm{PT}_{i}$ and $\mathrm{PR}_{i}$ on the $\mathrm{SU}$ average rate performance is demonstrated. More specifically, we model the noisy estimated version of $g_{i}$ using the well established model used in [23], [21]. Let 


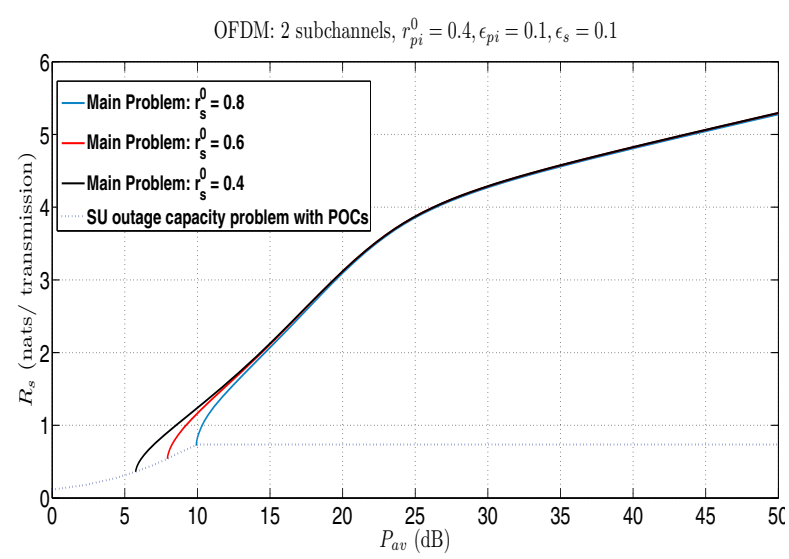

(a)

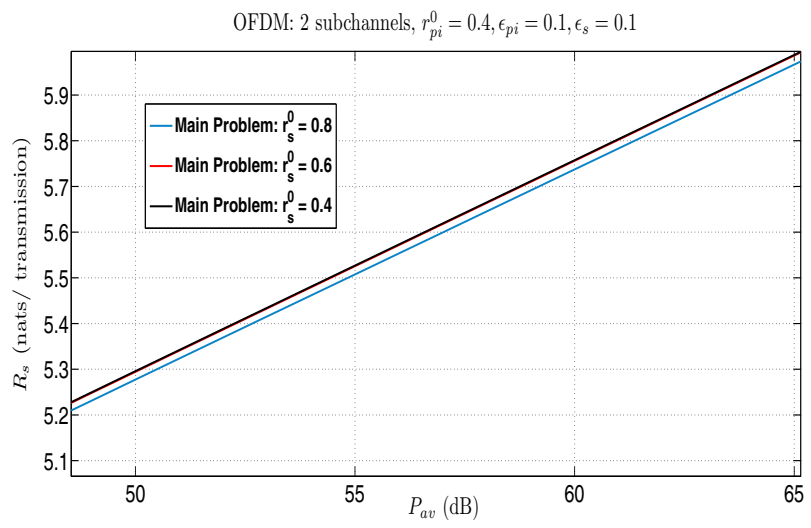

(b) Zooming the area of large $P_{a v}$

Fig. 5. Average SU rate performance from SU Ergodic capacity maximization problem with POC and SOC with varying $r_{s}^{0}\left(\epsilon_{p i}=0.1, \epsilon_{s}=0.1, r_{p i}^{0}=0.4\right.$

the complex channel amplitude gain between $\mathrm{PU}_{i}$ and $\mathrm{SU}$ be $a_{g, i}$. Thus, $g_{i}=\left|a_{g, i}\right|^{2}$, and the noisy estimated version of $a_{g, i}$ is modelled as $\hat{a}_{g, i}=\rho a_{g, i}+\sqrt{1-\rho^{2}} n_{i}$, where $0 \leq \rho \leq 1$ is the correlation coefficient between true and the estimated channel amplitude. $n_{i}$ is the estimation error of i-th subchannel which is distributed according to a complex normal distribution with zero mean and unit variance. Thus, the estimated channel power gain becomes $\hat{g}_{i}=\left|\hat{a}_{g, i}\right|^{2}$, which has an exponential distribution with unity mean identical to that of $g_{i}$. Figure 6 illustrates the effect of $\rho$ on the SU average rate when $N=2, r_{s}^{0}=0.6$ nats/transmission, $r_{p i}^{0}=0.4$ nats/transmission for all $i$, and the maximum primary and secondary outage probabilities are 0.1 . Note that in designing the power allocation policy, $\hat{g}_{i}$ is used instead of $g_{i}$, but the Lagrange multipliers are chosen such that the PU and SU outage constraints as well as the SU average power constraint are satisfied. Compared to the case of perfect knowledge of $g_{i}$ (i.e. $\rho=1$ ), the average rate loss for the SU is measured as approximately $6.4925 \%, 10.1505 \%$ and $20.3530 \%$ for $\rho=0.95,0.9$ and 0.8 , respectively for fixed $P_{a v}=15 \mathrm{~dB}$. (indicated by blue dashed line). This result indicates that, as $\rho$ decreases, the unreliability in estimated channel gain increases and thus places more restriction on the SU power policy in order to protect QoS in each primary link. Also, the SU requires more average power to make the

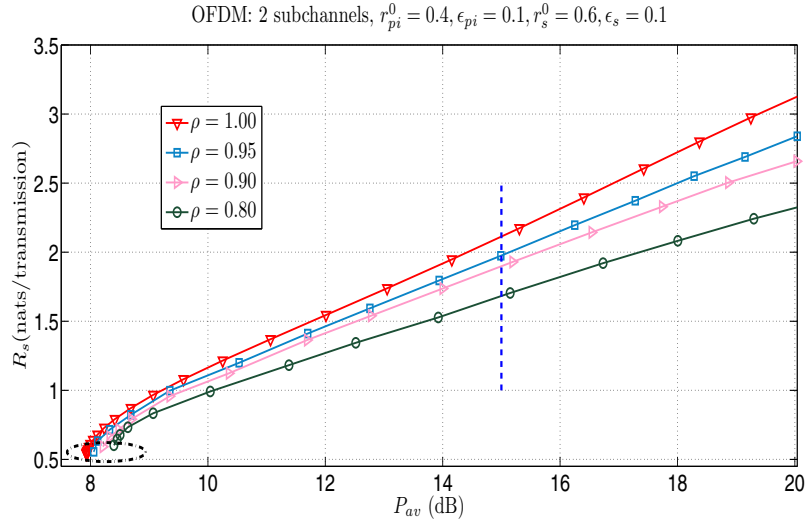

Fig. 6. The effect of the noisy primary channel estimates on SU ergodic capacity

problem feasible if $\rho$ decreases (indicated by the black dashed oval), since $\hat{g}_{i}$ also affects the term $\frac{h_{i}}{\alpha P_{p}\left(\hat{g}_{i}\right)+N_{0}}$. This results in the $\mathrm{SU}$ misinterpreting the ON-OFF status of $\mathrm{PU}_{i}$ and allocating incorrect power to the individual subchannels.

\section{Suboptimal power allocation scheme}

In this part we will illustrate the performance of the suboptimal power scheme in comparison with the optimal one. For these simulations, we use $N=16$ subchannels, $r_{s}^{0}=2$, $r_{p i}^{0}=1.25, \epsilon_{s}=\epsilon_{p i}=0.1$. In Fig. 7(a), it is seen that there is a small SU ergodic capacity loss of approximately $5.36 \%$ when the suboptimal algorithm is used, compared to the optimal power control policy, when $s_{a}^{*}$ and all $s_{i}^{*}$ is positive (Stage 2 mentioned above). However, our suboptimal power allocation policy is chosen carefully so that all POC and SOC are ensured, as illustrated in Fig. 7(b) for the same example. It implies that the SU can possibly sacrifice a small capacity loss by adopting this suboptimal power strategy, in return for a substantial reduction in real-time computational complexity while still ensuring that SOC and all POCs are satisfied.

\section{CONCLUSIONS}

In this paper, we have derived an optimal power allocation policy (under full CSI at the OFDM-based SU transmitter and receiver) in a spectrum sharing cognitive radio network over $N$ parallel fading channels, that maximizes SU ergodic capacity under a SU outage probability constraint, outage probability constraints on all $N$ primary users, and an average transmit power constraint at the SU transmitter. As a special case, we have also solved the secondary outage minimization problem when $N \geq 1$, thus generalizing previous work with $N=1$. The optimal solutions are derived by using a probabilistic power allocation technique that allows our results to be applicable to both continuous and discrete fading channels. To avoid an exponential computational complexity for deriving the SU's optimal power policy in real time for a given channel realization, we have proposed a low-complexity suboptimal power control policy, which substantially reduces the number of computations in each channel realization, while guaranteeing both PU's and SU's outage probability constraints for a small loss in the SU ergodic capacity performance. Numerical 


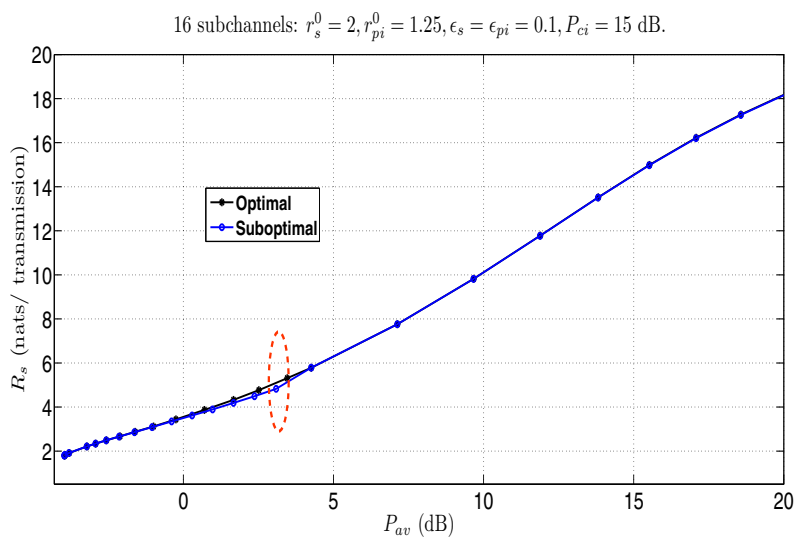

(a) SU capacity

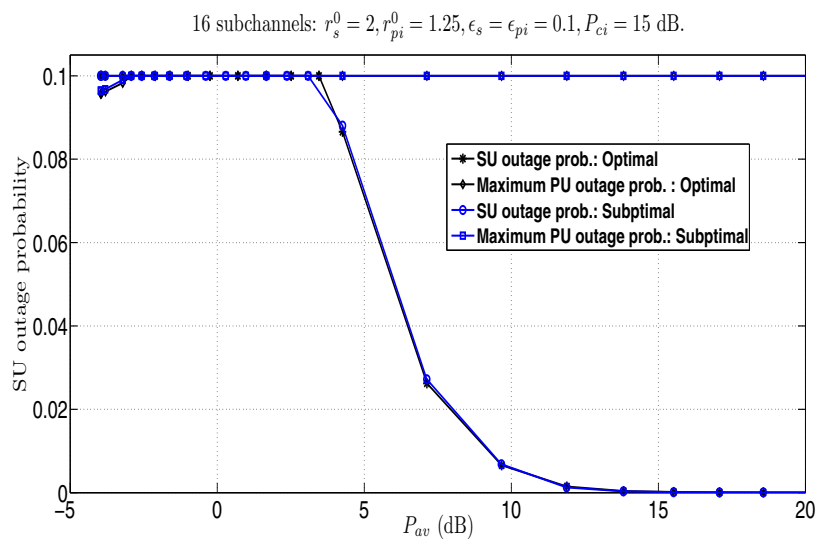

(b) Outage probability

Fig. 7. Performance comparison between optimal and proposed suboptimal power strategies $(N=16)$

results are presented to illustrate the performance of all derived algorithms.

\section{APPENDIX}

\section{A. Proof of Lemma 1}

We first show that for an arbitrary feasible probabilistic power scheme $\mathbf{P}_{s}(\boldsymbol{\nu})$, we can always construct another feasible scheme $\mathbf{P}_{s}^{\prime}(\boldsymbol{\nu})$ which is randomized among deterministic power schemes $p_{k i}(\boldsymbol{\nu})$ with time-sharing factors $w_{k i}(\boldsymbol{\nu})$, and performs equally well or better than $\mathbf{P}_{s}(\boldsymbol{\nu})$. Since $\mathbf{P}_{s}(\boldsymbol{\nu})$ is feasible, $\mathbf{P}_{s}(\boldsymbol{\nu})$ satisfies all the constraints, i.e. $E\left[\left\langle\boldsymbol{P}_{s i}(\boldsymbol{\nu})\right\rangle\right] \leq P_{a v}, \operatorname{Pr}\left\{r_{p i}\left(\boldsymbol{\nu}, P_{s i}(\boldsymbol{\nu})\right)<r_{p i}^{0}\right\} \leq \epsilon_{p i}$, and $\operatorname{Pr}\left\{\sum_{i=1}^{N} r_{s i}\left(\boldsymbol{\nu}, P_{s i}(\boldsymbol{\nu})\right)<r_{s}^{0}\right\} \leq \epsilon_{s}$. By the definitions of $p_{k i}(\boldsymbol{\nu})$ in (4) and $w_{k i}(\boldsymbol{\nu})$ in (3), we have the following condition:

$$
\begin{aligned}
r_{s}^{0} & \leq E\left[\sum_{i=1}^{N} r_{s i}\left(\boldsymbol{\nu}, P_{s i}(\boldsymbol{\nu})\right) \mid \sum_{i=1}^{N} r_{s i}\left(\boldsymbol{\nu}, p_{s i}(\boldsymbol{\nu})\right) \geq r_{s}^{0}, \boldsymbol{\nu}\right] \\
& \stackrel{(a)}{\leq} \sum_{i=1}^{N} r_{s i}\left(\boldsymbol{\nu}, E\left[P_{s i}(\boldsymbol{\nu}) \mid \sum_{i=1}^{N} r_{s i}\left(\boldsymbol{\nu}, p_{s i}(\boldsymbol{\nu})\right) \geq r_{s}^{0}, \boldsymbol{\nu}\right]\right) \\
& \stackrel{(b)}{=} \sum_{i=1}^{N} r_{s i}\left(\boldsymbol{\nu}, \frac{w_{1 i}(\boldsymbol{\nu})}{w_{a}(\boldsymbol{\nu})} p_{1 i}(\boldsymbol{\nu})+\frac{w_{a}(\boldsymbol{\nu})-w_{1 i}(\boldsymbol{\nu})}{w_{a}(\boldsymbol{\nu})} p_{2 i}(\boldsymbol{\nu})\right)
\end{aligned}
$$

where (a) follows from Jensen's inequality since $\sum_{i=1}^{N} r_{s i}\left(\boldsymbol{\nu}, p_{s i}(\boldsymbol{\nu})\right)$ is concave over $\boldsymbol{p}_{s}(\boldsymbol{\nu})$ and (b) follows from the fact that $E\left[P_{s i}(\boldsymbol{\nu}) \mid \sum_{i=1}^{N} r_{s i}\left(\boldsymbol{\nu}, p_{s i}(\boldsymbol{\nu})\right) \geq r_{s}^{0}, \boldsymbol{\nu}\right]=$ $\frac{w_{1 i}(\boldsymbol{\nu})}{w_{a}(\boldsymbol{\nu})} p_{1 i}(\boldsymbol{\nu})+\frac{w_{a}(\boldsymbol{\nu})-w_{1 i}(\boldsymbol{\nu})}{w_{a}(\boldsymbol{\nu})} p_{2 i}(\boldsymbol{\nu})$ by conditional expectation.

Furthermore, for all $i, \operatorname{Pr}\left\{P_{s i}^{\prime}(\boldsymbol{\nu})=p_{1 i}(\boldsymbol{\nu})\right\}=w_{1 i}(\boldsymbol{\nu})$ and $\operatorname{Pr}\left\{P_{s i}^{\prime}(\boldsymbol{\nu})=p_{2 i}(\boldsymbol{\nu})\right\}=w_{2 i}(\boldsymbol{\nu})$. Hence, $w_{1 i}(\boldsymbol{\nu})+w_{2 i}(\boldsymbol{\nu})=$ $\operatorname{Pr}\left\{P_{s i}^{\prime}(\boldsymbol{\nu})=E\left[P_{s i}(\boldsymbol{\nu}) \mid \sum_{i=1}^{N} r_{s i}\left(\boldsymbol{\nu}, p_{s i}(\boldsymbol{\nu})\right) \geq r_{s}^{0}, \boldsymbol{\nu}\right] \mid \boldsymbol{\nu}\right\}=$ $w_{a}(\boldsymbol{\nu})$ for all $i$. In other words,

$$
\operatorname{Pr}\left\{\mathbf{P}_{s}^{\prime}(\boldsymbol{\nu})=E\left[\mathbf{P}_{s}(\boldsymbol{\nu}) \mid \sum_{i=1}^{N} r_{s i}\left(\boldsymbol{\nu}, p_{s i}(\boldsymbol{\nu})\right) \geq r_{s}^{0}, \boldsymbol{\nu}\right] \mid \boldsymbol{\nu}\right\}=
$$
$w_{a}(\boldsymbol{\nu})$. Therefore, $\operatorname{Pr}\left\{\sum_{i=1}^{N} r_{s i}\left(\boldsymbol{\nu}, P_{s i}^{\prime}(\boldsymbol{\nu})\right) \geq r_{s}^{0} \mid \boldsymbol{\nu}\right\} \geq w_{a}(\boldsymbol{\nu})$ and $E\left[\operatorname{Pr}\left\{\sum_{i=1}^{N} r_{s i}\left(\boldsymbol{\nu}, P_{s i}^{\prime}(\boldsymbol{\nu})\right) \geq r_{s}^{0} \mid \boldsymbol{\nu}\right\}\right] \geq E\left[w_{a}(\boldsymbol{\nu})\right] \geq 1-\epsilon_{s}$. This implies that the new power scheme $\mathbf{P}_{s}^{\prime}(\boldsymbol{\nu})$, if feasible, results in an SU outage probability performance that will not be worse than that of $\mathbf{P}_{s}(\boldsymbol{\nu})$.

Now, we will show that $r_{p i}\left(\boldsymbol{\nu}, E\left[P_{s i}(\boldsymbol{\nu}) \mid r_{p i}\left(\boldsymbol{\nu}, p_{s i}(\boldsymbol{\nu})\right) \geq r_{p i}^{0}, \boldsymbol{\nu}\right]\right) \quad \geq \quad r_{p i}^{0}$. Note that when $r_{p i}\left(\boldsymbol{\nu}, p_{s i}(\boldsymbol{\nu})\right) \geq r_{p i}^{0}$, it implies $p_{s i}(\boldsymbol{\nu}) \leq \frac{1}{\beta_{i}}\left(\frac{g_{i} P_{p i}\left(g_{i}\right)}{e^{r_{p i}}-1}-N_{0}\right)^{+}$, i.e. the possible solution lies in a halfspace. Further, notice that $E\left[P_{s i}(\boldsymbol{\nu}) \mid r_{p i}\left(\boldsymbol{\nu}, p_{s i}(\boldsymbol{\nu})\right) \geq r_{p i}^{0}, \boldsymbol{\nu}\right]$ is a convex combination of the possible solutions in that halfspace weighted by the probability that each solution can happen. So, $E\left[P_{s i}(\boldsymbol{\nu}) \mid r_{p i}\left(\boldsymbol{\nu}, p_{s i}(\boldsymbol{\nu})\right) \geq r_{p i}^{0}, \boldsymbol{\nu}\right]$ also lies in the same halfspace due to convex set properties, which means

$$
\begin{aligned}
r_{p i}^{0} & \leq r_{p i}\left(\boldsymbol{\nu}, E\left[P_{s i}(\boldsymbol{\nu}) \mid r_{p i}\left(\boldsymbol{\nu}, p_{s i}(\boldsymbol{\nu})\right) \geq r_{p i}^{0}, \boldsymbol{\nu}\right]\right) \\
& \stackrel{(c)}{=} r_{p i}\left(\boldsymbol{\nu}, \frac{w_{1 i}(\boldsymbol{\nu})}{w_{1 i}(\boldsymbol{\nu})+w_{3 i}(\boldsymbol{\nu})} p_{1 i}(\boldsymbol{\nu})+\frac{w_{1 i}(\boldsymbol{\nu})}{w_{3 i}(\boldsymbol{\nu})+w_{3 i}(\boldsymbol{\nu})} p_{3 i}(\boldsymbol{\nu})\right)
\end{aligned}
$$

where $(c)$ follows again from the definition of conditional expectation. Also, $\operatorname{Pr}\left\{P_{s i}^{\prime}(\boldsymbol{\nu})=E\left[P_{s i}(\boldsymbol{\nu}) \mid r_{p i}\left(\boldsymbol{\nu}, p_{s i}(\boldsymbol{\nu})\right) \geq r_{p i}^{0}, \boldsymbol{\nu}\right] \mid \boldsymbol{\nu}\right\}=$ $w_{1 i}(\boldsymbol{\nu})+w_{3 i}(\boldsymbol{\nu})$ and it can be shown that $E\left[\operatorname{Pr}\left\{r_{p i}\left(\boldsymbol{\nu}, P_{s i}^{\prime}(\boldsymbol{\nu})\right) \geq r_{p i}^{0} \mid \boldsymbol{\nu}\right\}\right] \geq E\left[w_{1 i}(\boldsymbol{\nu})+w_{3 i}(\boldsymbol{\nu})\right] \geq$ $1-\epsilon_{p i}$.

Also, $E\left[\left\langle\boldsymbol{P}_{s}^{\prime}(\boldsymbol{\nu})\right\rangle\right]=E\left[\sum_{i=1}^{N} \sum_{k=1}^{4} w_{k i}(\boldsymbol{\nu}) p_{k i}(\boldsymbol{\nu})\right]=$ $E\left[\left\langle\boldsymbol{P}_{s}(\boldsymbol{\nu})\right\rangle\right]$. The feasibility of $\mathbf{P}^{\prime}{ }_{s}(\boldsymbol{\nu})$ has thus been proved. Finally, it can be shown that $E\left[\sum_{i=1}^{N} r_{s i}\left(\boldsymbol{\nu}, P_{s i}^{\prime}(\boldsymbol{\nu})\right)\right]=$ $E\left[\sum_{i=1}^{N} \sum_{k=1}^{4} w_{k i}(\boldsymbol{\nu}) r_{s i}\left(\boldsymbol{\nu}, p_{k i}(\boldsymbol{\nu})\right)\right] \geq E\left[\sum_{i=1}^{N} r_{s i}\left(\boldsymbol{\nu}, P_{s i}(\boldsymbol{\nu})\right)\right]$, where the inequality follows from Jensen's inequality. This completes the proof.

\section{B. Proof of convexity of (5)}

Here we suppress the dependence of the determistic power policies and the weighting functions on $\boldsymbol{\nu}$. Let $x_{k i}=w_{k i} p_{k i}$ where $w_{2 i}=w_{a}-w_{1 i}$. Then we can map $\left(p_{1 i}, p_{2 i}, p_{3 i}, p_{4 i}, w_{1 i}, w_{3 i}, w_{4 i}, w_{a}\right)$ to $\left(x_{1}, x_{2}, x_{3}, x_{4}, w_{1 i}, w_{3 i}, w_{4 i}, w_{a}\right)$, such that (5) can be rewritten as shown in (24). 


$$
\begin{array}{cl}
\max _{\mathscr{Z}\left(x_{k i}, w_{k i}, \forall k, \forall i\right)} & E\left[\sum_{i=1}^{N} \sum_{k=1}^{4} w_{k i} r_{s}\left(\boldsymbol{\nu}, \frac{x_{k i}}{w_{k i}}\right)\right] \\
\text { s.t. } & \text { (a) } E\left[\sum_{i=1}^{N} \sum_{k=1}^{4} x_{k i}\right] \leq P_{a v}, \\
& \text { (b) } E\left[w_{1 i}+w_{3 i}\right] \geq 1-\epsilon_{p i}, \\
& \text { (c) } E\left[w_{1 i}+w_{2 i}\right]=E\left[w_{a}\right] \geq 1-\epsilon_{s}, \\
& \text { (d) } \sum_{i=1}^{N} r_{s i}\left(\boldsymbol{\nu}, \frac{w_{1 i}}{w_{a}} \frac{x_{1 i}}{w_{1 i}}+\frac{\left(w_{a}-w_{1 i}\right)}{w_{a}} \frac{x_{2 i}}{w_{a}-w_{1 i}}\right)-r_{s}^{0} \geq 0 \\
& \text { (e) } w_{1 i} p_{r p, i}(\boldsymbol{\nu})+w_{3 i} p_{r p, i}(\boldsymbol{\nu})-x_{1 i}-x_{3 i} \geq 0, \quad \text { (f) } w_{a}-w_{1 i}=w_{2 i} \geq 0, \\
& \text { (g) } 1-w_{a}-w_{3 i}=w_{4 i} \geq 0, \quad(h) x_{k i} \geq 0, \quad \text { (i) } w_{k i} \geq 0,
\end{array}
$$

Note that in the objective function of (24), $w_{k i} r_{s}\left(\nu, \frac{x_{k i}}{w_{k i}}\right)$ is concave over $\left(x_{k i}, w_{k i}\right)$ (see [14]). To show that (24d) is convex, we can rearrange the inequality to be $\prod_{i=1}^{N}\left[w_{a}+\frac{h_{i}}{\alpha P_{p i}\left(g_{i}\right)+N_{0}}\left(x_{1 i}+x_{2 i}\right)\right]^{1 / N} \geq w_{a} e^{\frac{r_{s}^{0}}{N}}$. Since $t(z)=\left(\prod_{i=1}^{K} z\right)^{1 / N}$ is concave on $z \in \Re_{+}^{K}$ [24], the left side of the inequality is concave with respect to $\left(w_{a}, x_{1 i}, x_{2 i}\right)$, while the right hand side is linear over $w_{a}$. This results in a convex constraint when written in the standard form. The convexity of the rest of the constraints are obvious. This proves the convexity of (5).

\section{Proof of Theorem 1}

We will first solve for $p_{k i}^{*}(\boldsymbol{\nu})$ and then derive the solution for all optimal weighting functions. With $w_{k i}^{*}(\boldsymbol{\nu})=0$, the solution of $p_{k i}^{*}(\boldsymbol{\nu})$ has no impact on the optimization problem. Hence, we will consider the case $w_{k i}^{*}(\boldsymbol{\nu})>0$ in order to solve for $p_{k i}^{*}(\boldsymbol{\nu})$. Due to space limitations, we will show the derivation for $p_{1 i}^{*}(\boldsymbol{\nu})$ only and the finalized expressions for all $p_{k i}^{*}(\boldsymbol{\nu})$ for $k=2,3,4$.

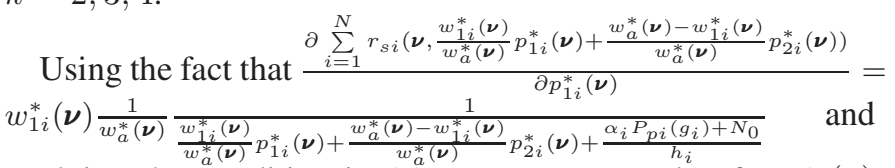
applying the condition in (7), we can now solve for $p_{1 i}^{*}(\boldsymbol{\nu})$ (when $w_{1 i}^{*}(\boldsymbol{\nu})>0$ ) as follows:

(1) When $q^{*}(\boldsymbol{\nu})=0$ and $u_{i}^{*}(\boldsymbol{\nu})=0: p_{1 i}^{*}(\boldsymbol{\nu})=p_{w f, i}\left(\boldsymbol{\nu}, \lambda^{*}\right)$. (2) When $q^{*}(\boldsymbol{\nu})=0$ and $u_{i}^{*}(\boldsymbol{\nu})>0$ : In this case, using the associated KKT conditions, we can show that the only possibility is $p_{1 i}^{*}(\boldsymbol{\nu})=p_{3 i}^{*}(\boldsymbol{\nu})=p_{r p, i}(\boldsymbol{\nu})$ if both $w_{1 i}^{*}(\boldsymbol{\nu})$ and $w_{3 i}^{*}(\boldsymbol{\nu})$ are positive. However, this is impossible due to the definitions of $p_{1 i}^{*}(\boldsymbol{\nu}), p_{3 i}^{*}(\boldsymbol{\nu})$.

(3) When $q^{*}(\boldsymbol{\nu})>0 \quad$ First, let $w_{1 i}^{*}(\boldsymbol{\nu})>0$ and $w_{a}^{*}(\boldsymbol{\nu})-w_{1 i}^{*}(\boldsymbol{\nu})>0$. Then one should have $\frac{w_{1 i}^{*}(\boldsymbol{\nu})}{w_{a}^{*}(\boldsymbol{\nu})} p_{1 i}^{*}(\boldsymbol{\nu})+\frac{w_{a}^{*}(\boldsymbol{\nu})-w_{1 i}^{*}(\boldsymbol{\nu})}{w_{a}^{*}(\boldsymbol{\nu})} p_{2 i}^{*}(\boldsymbol{\nu})=p_{r s, i}(\boldsymbol{\nu})$ in every $i$-th subchannel in order to achieve minimum power consumption. In this case, one can show after some analysis that the only possibility is $p_{1 i}^{*}(\boldsymbol{\nu})=p_{2 i}^{*}(\boldsymbol{\nu})=p_{r s, i}(\boldsymbol{\nu})$. However, this is impossible due to the definition in (4). Hence, to achieve $r_{s}^{0}$ with probability $w_{a}^{*}(\boldsymbol{\nu})$ for a given $\boldsymbol{\nu}$, it can be achieved by either (1) $w_{1 i}^{*}(\boldsymbol{\nu})=w_{a}^{*}(\boldsymbol{\nu}), w_{2 i}^{*}(\boldsymbol{\nu})=0, p_{1 i}^{*}(\boldsymbol{\nu})=p_{r s, i}(\boldsymbol{\nu})$, or (2) $w_{1 i}^{*}(\boldsymbol{\nu})=0, w_{2 i}^{*}(\boldsymbol{\nu})=w_{a}^{*}(\boldsymbol{\nu}), p_{2 i}^{*}(\boldsymbol{\nu})=p_{r s, i}(\boldsymbol{\nu})$.

Now, we will consider the two sub-cases when $u_{i}^{*}(\boldsymbol{\nu})>0$ and $u_{i}^{*}(\boldsymbol{\nu})=0$. When $q^{*}(\boldsymbol{\nu})>0$ and $u_{i}^{*}(\boldsymbol{\nu})>0$, we can show that since $u_{i}^{*}(\boldsymbol{\nu})>0, p_{1 i}^{*}(\boldsymbol{\nu})=p_{r p, i}$.
However, this optimal power $p_{1 i}^{*}(\boldsymbol{\nu})=p_{r p, i}$ is not one of the conditions for $q^{*}(\boldsymbol{\nu})>0$ discussed above, unless $p_{r p, i}=p_{r s, i}$ with positive probability, which is not possible for a continuous fading scenario. Although it is possible that $p_{r p, i}=p_{r s, i}$ for a discrete fading scenario, this will not affect the optimal solution. When $q^{*}(\boldsymbol{\nu})>0$ and $u_{i}^{*}(\boldsymbol{\nu})=0$, we have $0=\left[\frac{1}{p_{1 i}^{*}(\boldsymbol{\nu})+\frac{\alpha_{i} P_{p i}\left(g_{i}\right)+N_{0}}{h_{i}}}-\lambda^{*}\right]+$ $q^{*}(\boldsymbol{\nu})\left[\frac{1}{w_{a}^{*}(\boldsymbol{\nu})} \frac{1}{\frac{w_{i j}^{*}(\boldsymbol{\nu})}{w_{a}^{*}(\boldsymbol{\nu})} p_{1 i}^{*}(\boldsymbol{\nu})+\frac{w_{a}^{*}(\boldsymbol{\nu})-w_{1 i}^{*}(\boldsymbol{\nu})}{w_{a}^{*}(\boldsymbol{\nu})} p_{2 i}^{*}(\boldsymbol{\nu})+\frac{\alpha_{i} P_{p i}\left(g_{i}\right)+N_{0}}{h_{i}}}\right]$. We can now show via contradiction that if $q^{*}(\boldsymbol{\nu})>0$ and $w_{1 i}^{*}(\boldsymbol{\nu})>0$, then the only possibility is $p_{1 i}^{*}(\boldsymbol{\nu})=p_{r s, i}(\boldsymbol{\nu})$ while $w_{2 i}^{*}(\boldsymbol{\nu})=0$. Furthermore, it is impossible for $p_{1 i}^{*}(\boldsymbol{\nu})=p_{r s, i}(\boldsymbol{\nu})$ to hold when $\mathbf{p}_{r s}(\boldsymbol{\nu}) \prec \mathbf{p}_{w f}\left(\boldsymbol{\nu}, \lambda^{*}\right)$ because $p_{r s, i}^{*}(\boldsymbol{\nu})=\left(\frac{1+\frac{q^{*}(\boldsymbol{\nu})}{w_{*}^{*}(\boldsymbol{\nu})}}{\lambda^{*}}-\frac{\alpha_{i} P_{p i}\left(g_{i}\right)+N_{0}}{h_{i}}\right)^{+}>p_{w f, i}^{*}\left(\boldsymbol{\nu}, \lambda^{*}\right), \forall i$. Applying a similar procedure to $p_{k i}^{*}(\boldsymbol{\nu})$, for $k=2,3,4$, we obtain the closed form solutions of all $p_{k i}^{*}(\boldsymbol{\nu})$ as follows

$$
\begin{aligned}
& p_{1 i}^{*}(\boldsymbol{\nu})=\left\{\begin{array}{lc}
p_{w f, i}\left(\boldsymbol{\nu}, \lambda^{*}\right), & w_{1 i}^{*}(\boldsymbol{\nu})>0, \mathbf{p}_{r s}(\boldsymbol{\nu}) \preceq \mathbf{p}_{w f}\left(\boldsymbol{\nu}, \lambda^{*}\right), \\
& p_{w f, i}\left(\boldsymbol{\nu}, \lambda^{*} \leq p_{r p, i}(\boldsymbol{\nu})\right. \\
p_{r s, i}(\boldsymbol{\nu}), & w_{1 i}^{*}(\boldsymbol{\nu})>0, \mathbf{p}_{w f}\left(\boldsymbol{\nu}, \lambda^{*}\right) \prec \mathbf{p}_{r s}(\boldsymbol{\nu}), \\
& p_{w f, i}\left(\boldsymbol{\nu}, \lambda^{*} \leq p_{r p, i}(\boldsymbol{\nu})\right. \\
p_{r p, i}(\boldsymbol{\nu}), & w_{1 i}^{*}(\boldsymbol{\nu})>0, \mathbf{p}_{r s}(\boldsymbol{\nu}) \preceq \mathbf{p}_{w f}\left(\boldsymbol{\nu}, \lambda^{*}\right), \\
& p_{w f, i}\left(\boldsymbol{\nu}, \lambda^{*}\right)>p_{r p, i}(\boldsymbol{\nu})
\end{array}\right. \\
& p_{2 i}^{*}(\boldsymbol{\nu})=\left\{\begin{array}{lc}
p_{w f, i}\left(\boldsymbol{\nu}, \lambda^{*}\right), & w_{a}^{*}(\boldsymbol{\nu})-w_{1 i}^{*}(\boldsymbol{\nu})>0, \\
& \mathbf{p}_{r s}(\boldsymbol{\nu}) \preceq \mathbf{p}_{w f}\left(\boldsymbol{\nu}, \lambda^{*}\right) \\
p_{r s, i}(\boldsymbol{\nu}), & w_{a}^{*}(\boldsymbol{\nu})-w_{1 i}^{*}(\boldsymbol{\nu})>0, \\
& \mathbf{p}_{w f}\left(\boldsymbol{\nu}, \lambda^{*}\right) \prec \mathbf{p}_{r s}(\boldsymbol{\nu})
\end{array}\right. \\
& p_{3 i}^{*}(\boldsymbol{\nu})= \begin{cases}p_{w f, i}\left(\boldsymbol{\nu}, \lambda^{*}\right), & w_{3 i}^{*}(\boldsymbol{\nu})>0, p_{w f, i}\left(\boldsymbol{\nu}, \lambda^{*}\right) \leq p_{r p, i}(\boldsymbol{\nu}) \\
p_{r p, i}(\boldsymbol{\nu}), & w_{3 i}^{*}(\boldsymbol{\nu})>0, p_{w f, i}\left(\boldsymbol{\nu}, \lambda^{*}\right)>p_{r p, i}(\boldsymbol{\nu})\end{cases} \\
& p_{4 i}^{*}(\boldsymbol{\nu})=p_{w f, i}\left(\boldsymbol{\nu}, \lambda^{*}\right), \quad w_{4 i}^{*}(\boldsymbol{\nu})>0
\end{aligned}
$$

Now, we will derive optimal solutions for $w_{1 i}^{*}(\boldsymbol{\nu}), w_{3 i}^{*}(\boldsymbol{\nu})$, and $w_{a}^{*}(\boldsymbol{\nu})$.

Optimal solution for $w_{3 i}^{*}(\boldsymbol{\nu}):$ It is obvious that when $1-$ $w_{a}^{*}(\boldsymbol{\nu})=0, w_{3 i}^{*}(\boldsymbol{\nu})=w_{4 i}^{*}(\boldsymbol{\nu})=0$. When $1-w_{a}^{*}(\boldsymbol{\nu})>0$, it follows easily from the KKT conditions and the definitions of the benefit functions (20) that (regardless of whether $q^{*}(\boldsymbol{\nu})$ is zero or strictly positive)

$$
w_{3 i}^{*}(\boldsymbol{\nu})= \begin{cases}0, & 1-w_{a}^{*}(\boldsymbol{\nu})>0, \mathscr{B}_{3 i, \boldsymbol{\nu}} \leq \mathscr{B}_{4 i, \boldsymbol{\nu}} \\ \kappa_{3 i}^{*}(\boldsymbol{\nu}), & 1-w_{a}^{*}(\boldsymbol{\nu})>0, \mathscr{B}_{3 i, \boldsymbol{\nu}}=\mathscr{B}_{4 i, \boldsymbol{\nu}} \\ 1-w_{a}^{*}(\boldsymbol{\nu}), & 1-w_{a}^{*}(\boldsymbol{\nu})>0, \mathscr{B}_{3 i, \boldsymbol{\nu}} \geq \mathscr{B}_{4 i, \boldsymbol{\nu}} \\ & w_{4 i}^{*}(\boldsymbol{\nu})=\left(1-w_{a}^{*}(\boldsymbol{\nu})\right)-w_{3 i}^{*}(\boldsymbol{\nu}) .\end{cases}
$$

where $0<\kappa_{3 i}^{*}(\boldsymbol{\nu})<1-w_{a}^{*}(\boldsymbol{\nu})$. 
Optimal solutions for $w_{1 i}^{*}(\boldsymbol{\nu})$ and $w_{a}^{*}(\boldsymbol{\nu})$ : Here we use KKT condition (9) and

$$
\begin{aligned}
& \frac{\partial l(\ldots)}{\partial w_{1 i}^{*}(\boldsymbol{\nu})} \\
& =\left(s_{i}^{*}+\left(r_{s i}\left(\boldsymbol{\nu}, p_{1 i}^{*}(\boldsymbol{\nu})\right)-\lambda^{*} p_{1 i}^{*}(\boldsymbol{\nu})\right)\right) \\
& +\frac{q^{*}(\boldsymbol{\nu})}{w_{a}^{*}(\boldsymbol{\nu})} \frac{p_{1 i}^{*}(\boldsymbol{\nu})-p_{2 i}^{*}(\boldsymbol{\nu})}{\frac{w_{1 i}^{*}(\boldsymbol{\nu})}{w_{a}^{*}(\boldsymbol{\nu})} p_{1 i}^{*}(\boldsymbol{\nu})+\frac{\left(w_{a}^{*}(\boldsymbol{\nu})-w_{1 i}^{*}(\boldsymbol{\nu})\right)}{w_{a}^{*}(\boldsymbol{\nu})} p_{2 i}^{*}(\boldsymbol{\nu})+\frac{\alpha_{i} P_{p i}(\boldsymbol{\nu})+N_{0}}{h_{i}}} \\
& -\left(r_{s i}\left(\boldsymbol{\nu}, p_{2 i}^{*}(\boldsymbol{\nu})\right)-\lambda^{*} p_{2 i}^{*}(\boldsymbol{\nu})+\gamma_{i}^{*}(\boldsymbol{\nu})\right)
\end{aligned}
$$

where note that the term $u_{i}^{*}(\boldsymbol{\nu})\left(p_{r p, i}(\boldsymbol{\nu})-p_{1 i}^{*}\right)=0$ due to the optimality condition. Similarly, from KKT condition (8), we also have

$$
\begin{aligned}
& \sum_{i=1}^{N}\left(r_{s i}\left(\boldsymbol{\nu}, p_{4 i}^{*}(\boldsymbol{\nu})\right)-\lambda^{*} p_{4 i}^{*}(\boldsymbol{\nu})+\eta_{i}^{*}(\boldsymbol{\nu})\right) \\
& =s_{a}^{*}+\sum_{i=1}^{N}\left(r_{s i}\left(\boldsymbol{\nu}, p_{2 i}^{*}(\boldsymbol{\nu})\right)-\lambda^{*} p_{2 i}^{*}(\boldsymbol{\nu})+\gamma_{i}^{*}(\boldsymbol{\nu})\right) \\
& -\frac{q^{*}(\boldsymbol{\nu}) w_{1 i}^{*}(\boldsymbol{\nu})}{\left(w_{a}^{*}(\boldsymbol{\nu})\right)^{2}} \frac{p_{1 i}^{*}(\boldsymbol{\nu})-p_{2 i}^{*}(\boldsymbol{\nu})}{\frac{w_{1 i}^{*}(\boldsymbol{\nu})}{w_{a}^{*}(\boldsymbol{\nu})} p_{1 i}^{*}(\boldsymbol{\nu})+\frac{\left(w_{a}^{*}(\boldsymbol{\nu})-w_{1 i}^{*}(\boldsymbol{\nu})\right)}{w_{a}^{*}(\boldsymbol{\nu})} p_{2 i}^{*}(\boldsymbol{\nu})+\frac{\alpha_{i} P_{p i}(\boldsymbol{\nu})+N_{0}}{h_{i}}}
\end{aligned}
$$

If $q^{*}(\boldsymbol{\nu})>0$, it can be shown that both $w_{1 i}^{*}(\boldsymbol{\nu})$ and $w_{2 i}^{*}(\boldsymbol{\nu})$ cannot be positive at the same time (i.e. $\mathscr{S}_{1} \cap \mathscr{S}_{2}$ is an empty set), and we have the two following cases: (1) $w_{1 i}^{*}(\boldsymbol{\nu})=$ $w_{a}^{*}(\boldsymbol{\nu})>0$ and $w_{2 i}^{*}(\boldsymbol{\nu})=0 \Rightarrow \gamma_{i}^{*}(\boldsymbol{\nu}) \geq 0$. In this case we have $p_{1 i}^{*}(\boldsymbol{\nu})=p_{r s, i}(\boldsymbol{\nu})$ and by arbitrarily choosing $p_{2 i}^{*}(\boldsymbol{\nu})=p_{r s, i}^{*}(\boldsymbol{\nu})$ (since $w_{2 i}^{*}(\boldsymbol{\nu})=0$ ), one can show that $\mathscr{B}_{1 i, \boldsymbol{\nu}}=\mathscr{B}_{2 i, \boldsymbol{\nu}}+\gamma_{i}^{*}(\boldsymbol{\nu}) \geq \mathscr{B}_{2 i, \boldsymbol{\nu}}$. (2) $w_{1 i}^{*}(\boldsymbol{\nu})=0$ and $w_{2 i}^{*}(\boldsymbol{\nu})=w_{a}^{*}(\boldsymbol{\nu})>0 \Rightarrow \gamma_{i}^{*}(\boldsymbol{\nu})=0$. In this case one can show that (again by arbitrarily setting $p_{1 i}^{*}(\boldsymbol{\nu})=p_{2 i}^{*}(\boldsymbol{\nu})$ since $\left.w_{1 i}^{*}(\boldsymbol{\nu})=0\right) \mathscr{B}_{1 i, \boldsymbol{\nu}} \leq \mathscr{B}_{2 i, \boldsymbol{\nu}}$. Note also that when $w_{a}^{*}(\boldsymbol{\nu})=0$, obviously $w_{1 i}^{*}(\boldsymbol{\nu})=w_{2 i}^{*}(\boldsymbol{\nu})=0$.

Next, we separately consider the cases $q^{*}(\boldsymbol{\nu})>0$ and $q^{*}(\boldsymbol{\nu})=0$, and use the two specific scenarios $0<w_{a}^{*}(\boldsymbol{\nu})<1$ and $w_{a}^{*}(\boldsymbol{\nu})=1$. Considering all possible combinations of these scenarios, and using the corresponding optimality conditions given by (8), (9), we can finally arrive at the following solutions for $w_{1 i}^{*}(\boldsymbol{\nu})$ and $w_{a}^{*}(\boldsymbol{\nu})$ (details can be found in [22]). When $q^{*}(\boldsymbol{\nu})>0$, we have

$$
w_{1 i}^{*}(\boldsymbol{\nu})= \begin{cases}0, & w_{a}^{*}(\boldsymbol{\nu})>0, \mathscr{B}_{1 i, \boldsymbol{\nu}} \leq \mathscr{B}_{2 i, \boldsymbol{\nu}} \\ w_{a}^{*}(\boldsymbol{\nu}), & w_{a}^{*}(\boldsymbol{\nu})>0, \mathscr{B}_{1 i, \boldsymbol{\nu}} \geq \mathscr{B}_{2 i, \boldsymbol{\nu}}\end{cases}
$$

When $q^{*}(\boldsymbol{\nu})=0$, we have

$$
w_{1 i}^{*}(\boldsymbol{\nu})= \begin{cases}0, & w_{a}^{*}(\boldsymbol{\nu})>0, \mathscr{B}_{1 i, \boldsymbol{\nu}} \leq \mathscr{B}_{2 i, \boldsymbol{\nu}} \\ \kappa_{1 i}^{*}(\boldsymbol{\nu}), & w_{a}^{*}(\boldsymbol{\nu})>0, \mathscr{B}_{1 i, \boldsymbol{\nu}}=\mathscr{B}_{2 i, \boldsymbol{\nu}} \\ w_{a}^{*}(\boldsymbol{\nu}), & w_{a}^{*}(\boldsymbol{\nu})>0, \mathscr{B}_{1 i, \boldsymbol{\nu}} \geq \mathscr{B}_{2 i, \boldsymbol{\nu}}\end{cases}
$$

where $0<\kappa_{1 i}^{*}(\boldsymbol{\nu})<w_{a}^{*}(\boldsymbol{\nu})$, and $w_{2 i}^{*}(\boldsymbol{\nu})=w_{a}^{*}(\boldsymbol{\nu})-w_{1 i}^{*}(\boldsymbol{\nu})$ for both cases.

Finally, regardless of whether we have $q^{*}(\boldsymbol{\nu})>0$ or $q^{*}(\boldsymbol{\nu})=0$, we can show that the optimal solution of $w_{a}^{*}(\boldsymbol{\nu})$ is defined in (29).

Also, note that for the continuous fading case, (29) simplifies and $w_{a}^{*}(\boldsymbol{\nu})$ is either 0 or 1 .

\section{REFERENCES}

[1] "Spectrum policy task force," Federal Communications Commission (FCC), Rep. ET Docket no. 02-135, Tech. Rep., Nov. 2002.

[2] J. Mitola, "Cognitive radio: an integrated agent architecture for software defined radio," Ph.D. dissertation, KTH, Stockholm, Sweden, Dec. 2000.

[3] A. Goldsmith, S. Jafar, I. Marić, and S. Srinivasa, "Breaking spectrum gridlock with cognitive radios: an information theoretic perspective," Proc. IEEE, vol. 97, no. 5, pp. 894-914, May 2009.

[4] T. Weiss and F. Jondral, "Spectrum pooling: an innovative strategy for the enhancement of spectrum efficiency," IEEE Commun. Mag., vol. 42, vol. 42, no. 3, pp. S8-S14, Mar. 2004

[5] G. Bansal, J. Hossain, and V. Bhargava, "Optimal and suboptimal power allocation schemes for OFDM-based cognitive radio systems," IEEE Trans. Wireless Commun., vol. 7, no. 11, pp. 4710-4718, Nov. 2008.

[6] P. Wang, M. Zhao, L. Xiao, S. Zhou, and J. Wang, "Power allocation in OFDM-based cognitive radio systems," in Proc. 2007 Global Telecommunications Conference, pp. 4061-4065.

[7] X. Kang, H. Garg, Y.-C. Liang, and R. Zhang, "Optimal power allocation for OFDM-based cognitive radio with new primary transmission protection criteria," IEEE Trans. Wireless Commun., vol. 9, no. 6, pp. 2066-2075, 2010.

[8] D. Tse and P. Viswanath, Fundamentals of Wireless Communication. Cambridge University Press, 2005.

[9] J. Jang and K. B. Lee, "Transmit power adaptation for multiuser OFDM systems," IEEE J. Sel. Areas Commun., vol. 2, no. 2, pp. 171-178, Feb. 2003.

[10] X. Kang, R. Zhang, Y.-C. Liang, and H. K. Garg, "Optimal power allocation for cognitive radio under primary user's outage loss constraint," in Proc. 2009 IEEE International Conference on Communications, pp. $1-5$.

[11] — " "On outage capacity of secondary users in fading cognitive radio networks with primary users outage constraint," in Proc. 2009 IEEE GLOBECOM, pp. 1-5.

[12] X. Kang, R. Zhang, Y.-C. Liang, and H. Garg, "Optimal power allocation strategies for fading cognitive radio channels with primary user outage constraint," IEEE J. Sel. Areas Commun., vol. 29, no. 2, pp. 374-383, Feb. 2011

[13] L. Musavian, S. Aïssa, and S. Lambotharan, "Adaptive modulation in spectrum-sharing channels under delay quality-of-service constraints," IEEE Trans. Veh. Technol., vol. 60, no. 3, pp. 901-911, Mar. 2011.

[14] J. Luo, R.Yates, and P. Spasojevic, "Service outage based power and rate allocation for parallel fading channels," IEEE Trans. Inf. Theory, vol. 51, no. 7, pp. 2594-2611, July 2005.

[15] A. Limmanee, S.Dey, and J. Evans, "Service-outage capacity maximization in cognitive radio," accepted by the 2011 IEEE International Conference on Communications.

[16] G. Caire, G. Taricco, and E. Biglieri, "Optimum power control over fading channels," IEEE Trans. Inf. Theory, vol. 5, pp. 1468-1489, 1999.

[17] R. Zhang, "Optimal power control over fading cognitive radio channels by exploiting primary user CSI," in Proc. 2008 IEEE GLOBECOM, pp. $1-5$.

[18] K. Eswaran, M. Gastpar, and K. Ramchandran, "Bits through ARQs spectrum sharing with a primary packet system," in Proc. 2007 IEEE International Symposium on Information Theory, pp. 2171-2175.

[19] G. Ganesan and Y. Li, "Cooperative spectrum sensing in cognitive radio networks," in Proc. 2005 IEEE International Symposium on New Front. Dynamic Spectrum Access Networks, pp. 137-143.

[20] Y. He and S. Dey, "Power allocation in spectrum sharing cognitive radio networks with quantized channel information," IEEE Trans. Commun., vol. 59, no. 6, pp. 1644-1656, June 2011.

[21] _ _ "Throughput maximization in cognitive radio under peak interference constraints with limited feedback," IEEE Trans. Veh. Technol., vol. 9, pp. 1287-1305, Feb. 2012.

[22] A. Limmanee, S. Dey, and J. Evans, "Service-outage capacity maximization in cognitive radio for parallel fading channels," 2010. Available: http://www.cubinlab.ee.unimelb.edu.au/ athipatl/documents/ ServiceOutageCognitiveOFDM.pdf.

[23] K. Ahn and R. W. Heath, "Performance analysis of maximum ratio combining with imperfect channel estimation in the presence of cochannel interferences," IEEE Trans. Wireless Commun., vol. 8, no. 8, pp. 10801085, Mar. 2009.

[24] S. Boyd and L. Vandenberghe, Convex Optimization. Cambridge University Press, 2004

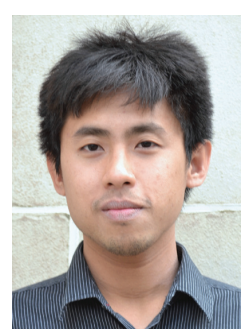

communications.
Athipat Limmanee (S'11) received the B.Eng. (with first-class honours) in communication engineering from Sirindhorn International Institute of Technology, Thammasat University, Thailand, in 2004. He is a recipient of the Melbourne International Research Scholarship and works towards his $\mathrm{Ph} . \mathrm{D}$. degree in Electrical and Electronic Engineering Department at The University of Melbourne, Australia. His research interests include cognitive radio networks, resource allocation in wireless networks, information theory and signal processing for 


$$
w_{a}^{*}(\boldsymbol{\nu})=\left\{\begin{aligned}
0, & s_{a}^{*}+\sum_{i \in \mathscr{S}_{1}} \mathscr{B}_{1 i, \boldsymbol{\nu}}+\sum_{i \in \mathscr{S}_{2}} \mathscr{B}_{2 i, \boldsymbol{\nu}}-\sum_{i \in \mathscr{S}_{1} \cap \mathscr{S}_{2}} \mathscr{B}_{1 i, \boldsymbol{\nu}} \\
& \leq \sum_{i \in \mathscr{S}_{3}} \mathscr{B}_{3 i, \boldsymbol{\nu}}+\sum_{i \in \mathscr{S}_{4}} \mathscr{B}_{4 i, \boldsymbol{\nu}}-\sum_{i \in \mathscr{S}_{3} \cap \mathscr{S}_{4}} \mathscr{B}_{3 i, \boldsymbol{\nu}} \\
\kappa_{a}^{*}(\boldsymbol{\nu}), s_{a}^{*} & +\sum_{i \in \mathscr{S}_{1}} \mathscr{B}_{1 i, \boldsymbol{\nu}}+\sum_{i \in \mathscr{S}_{2}} \mathscr{B}_{2 i, \boldsymbol{\nu}}-\sum_{i \in \mathscr{S}_{1} \cap \mathscr{S}_{2}} \mathscr{B}_{1 i, \boldsymbol{\nu}} \\
& =\sum_{i \in \mathscr{S}_{3}} \mathscr{B}_{3 i, \boldsymbol{\nu}}+\sum_{i \in \mathscr{S}_{4}} \mathscr{B}_{4 i, \boldsymbol{\nu}}-\sum_{i \in \mathscr{S}_{3} \cap \mathscr{S}_{4}} \mathscr{B}_{3 i, \boldsymbol{\nu}} \\
1, \quad s_{a}^{*} & +\sum_{i \in \mathscr{S}_{1}} \mathscr{B}_{1 i, \boldsymbol{\nu}}+\sum_{i \in \mathscr{S}_{2}} \mathscr{B}_{2 i, \boldsymbol{\nu}}-\sum_{i \in \mathscr{S}_{1} \cap \mathscr{S}_{2}} \mathscr{B}_{1 i, \boldsymbol{\nu}} \\
& \geq \sum_{i \in \mathscr{S}_{3}} \mathscr{B}_{3 i, \boldsymbol{\nu}}+\sum_{i \in \mathscr{S}_{4}} \mathscr{B}_{4 i, \boldsymbol{\nu}}-\sum_{i \in \mathscr{S}_{3} \cap \mathscr{S}_{4}} \mathscr{B}_{3 i, \boldsymbol{\nu}}
\end{aligned}\right.
$$

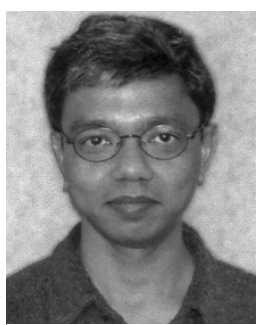

Subhrakanti Dey (SM'06) was born in India, in 1968. He received the B.Tech. and M.Tech. degrees from the Department of Electronics and Electrical Communication Engineering, Indian Institute of Technology, Kharagpur, India, in 1991 and 1993, respectively, and the Ph.D. degree from the Department of Systems Engineering, Research School of Information Sciences and Engineering, Australian National University, Canberra, Australia, in 1996. He has been with the Department of Electrical and Electronic Engineering, University of Melbourne, Australia , since February 2000, where he is currently a full Professor. From September 1995 to September 1997 and September 1998 to February 2000, he was a postdoctoral Research Fellow with the Department of Systems Engineering, Australian National University. From September 1997 to September 1998, he was a post-doctoral Research Associate with the Institute for Systems Research, University of Maryland, College Park. His current research interests include networked control systems, wireless communications and networks, signal processing for sensor networks, and stochastic and adaptive estimation and control. Prof. Dey currently serves on the Editorial Board of Elsevier Systems and Control Letters. He was also an Associate Editor for the IEEE Transactions on Signal Processing and the IEEE Transactions on Automatic Control. He is a Senior Member of IEEE.

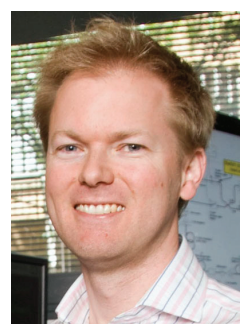

Jamie Evans was born in Newcastle, Australia, in 1970. He received the B.S. degree in physics and the B.E. degree in computer engineering from the University of Newcastle, in 1992 and 1993, respectively, where he received the University Medal upon graduation. He received the M.S. and the Ph.D. degrees from the University of Melbourne, Australia, in 1996 and 1998, respectively, both in electrical engineering, and was awarded the Chancellor's Prize for excellence for his Ph.D. thesis. From March 1998 to June 1999, he was a Visiting Researcher in the Department of Electrical Engineering and Computer Science, University of California, Berkeley. He returned to Australia to take up a position as Lecturer at the University of Sydney, Australia, where he stayed until July 2001. From July 2001 until March 2012 he was with the Department of Electrical and Electronic Engineering, University of Melbourne. He is currently a Professor in the Department of Electrical and Computer Systems Engineering at Monash University, Australia. His research interests are in communications theory, information theory, and statistical signal processing with a focus on wireless communications networks. 